\title{
Herz-Morrey-Hardy Spaces with Variable Exponents and Their Applications
}

\author{
Jingshi Xu and Xiaodi Yang \\ Department of Mathematics, Hainan Normal University, Haikou 571158, China \\ Correspondence should be addressed to Jingshi Xu; jingshixu@126.com
}

Received 5 July 2014; Accepted 20 December 2014

Academic Editor: Józef Banaś

Copyright (C) $2015 \mathrm{~J}$. Xu and X. Yang. This is an open access article distributed under the Creative Commons Attribution License, which permits unrestricted use, distribution, and reproduction in any medium, provided the original work is properly cited.

\begin{abstract}
The authors introduce Herz-Morrey-Hardy spaces with variable exponents and establish the characterization of these spaces in terms of atom. Applying the characterization, the authors obtain the boundedness of some singular integral operators on these spaces.
\end{abstract}

\section{Introduction}

The Herz spaces go back to Beurling and Herz; see $[1,2]$. Firstly, they attracted a lot of authors' attention because they could be used to characterize Fourier multipliers for Hardy spaces; see [3]. Then, in 1989 Chen and Lau in [4] and GarcíaCuerva in [5] introduced now called nonhomogeuous Herz type Hardy spaces. They found that these Herz type Hardy spaces have a decomposition via central atoms. After that, $\mathrm{Lu}$ et al. considered homogeuous Herz type Hardy spaces and also obtained a central atomic decomposition for them. Since then Herz type spaces have been studied extensively; see monograph [6] for details. Meanwhile, in the last three decades, the interest of the study for variable exponent spaces has been increasing year by year. Variable exponent spaces have many applications: in electrorheological fluid [7], in differential equations [8] and references therein, and in image restoration [9-11], for instance. Indeed, many spaces with variable exponents appeared, such as: Lebesgue spaces, Sobolev spaces and Bessel potential spaces with variable exponent, Besov and Triebel-Lizorkin spaces with variable exponents, Morrey spaces with variable exponents, Campanato spaces with variable exponent, and Hardy spaces with variable exponent; see [12-23] and references therein. Moreover, the atomic, molecular, and wavelet decompositions of variable exponent Besov and Triebel-Lizorkin spaces were given in $[13,14,20,21,24]$. The duality and reflexivity of spaces $B_{p(\cdot), q}^{s}$ and $F_{p(\cdot), q}^{s}$ were discussed in [25]. The atomic and molecular decompositions of Hardy spaces with variable exponent and their applications for the boundedness of singular integral operators were obtained in $[22,26]$.

Recently, as a generalization of Lebesgue spaces with variable exponent, Herz spaces with variable exponents are introduced. In fact, in 2010 Izuki proved the boundedness of sublinear operators on Herz space with variable exponents $\dot{K}_{p(\cdot)}^{\alpha, q}$ and $K_{p(\cdot)}^{\alpha, q}$ in [27]. In 2012, Almeida and Drihem obtained boundedness results for a wide class of classical operators on Herz spaces $\dot{K}_{p(\cdot)}^{\alpha(\cdot),}$ and $K_{p(\cdot)}^{\alpha(\cdot), q}$ in [28]. Shi and the first author in [29] considered Herz type Besov and TriebelLizorkin spaces with one variable exponent. Then Dong and first author in [30] established the boundedness of vectorvalued Hardy-Littlewood maximal operator in spaces $\dot{K}_{p(\cdot)}^{\alpha(\cdot), q}$ and $K_{p(\cdot)}^{\alpha(\cdot), q}$ and gave characterizations of Herz type Besov and Triebel-Lizorkin spaces with variable exponents by maximal functions. In [31], Wang and Liu introduced a certain Herz type Hardy spaces with variable exponent. In 2013, Samko introduced Herz spaces with three variable exponents and obtained the boundedness of Hardy-Littlewood maximal operator on them. In [32-34], the boundedness of singular integrals and their commutators of BMO functions are discussed in Herz Morrey spaces with variable exponents. The Herz-Morrey spaces with constants were considered in $[35,36]$; however, there is no theory of Herz-Morrey type Hardy spaces. In this paper we fill the gap and introduce HerzMorrey-Hardy spaces with variable exponents. 
The outline of the paper is as follows. In the rest of the section we will recall some definitions and notions. In Section 2, we will define the Herz-Morrey-Hardy spaces with variable exponents $H M \dot{K}_{p(\cdot), \lambda}^{\alpha(\cdot), q}$ and $H M K_{p(\cdot), \lambda}^{\alpha(\cdot), q}$ and give their atomic characterization. In Section 3, we obtain that certain singular integral operators are bounded from Herz-MorreyHardy spaces with variable exponents into Herz-Morrey spaces with variable exponents as an application of the atomic characterization.

Throughout this paper $|E|$ denotes the Lebesgue measure and $\chi_{E}$ the characteristic function for a measurable set $E \subset$ $\mathbb{R}^{n}$. For a multi-index $\beta=\left(\beta_{1}, \beta_{2}, \ldots, \beta_{n}\right)$, we denote $|\beta|=$ $\beta_{1}+\beta_{2}+\cdots+\beta_{n}$. We also use the notation $a \lesssim b$ if there exists a constant $c>0$ such that $a \leqslant c b$. If $a \leqslant b$ and $b \leqslant a$ we will write $a \approx b$. Finally we claim that $C$ is always a positive constant but it may change from line to line.

Definition 1. Let $E$ be a measurable set in $\mathbb{R}^{n}$ with $|E|>0$. Let $p(\cdot): E \rightarrow[1, \infty)$ be a measurable function. Denote

$$
L^{p(\cdot)}(E):=\left\{f \text { is measurable on } E: \rho_{p(\cdot)}\left(\frac{f}{\lambda}\right)<\infty\right.
$$

$$
\text { for some constant } \lambda>0\} \text {, }
$$

where $\rho_{p(\cdot)}(f):=\int_{E}|f(x)|^{p(x)} \mathrm{d} x$, and

$$
\|f\|_{L^{p(\cdot)}(E)}:=\inf \left\{\lambda>0: \rho_{p(\cdot)}\left(\frac{f}{\lambda}\right) \leqslant 1\right\} .
$$

Then $L^{p(\cdot)}(E)$ is a Banach space with the norm $\|\cdot\|_{L^{p(\cdot)}(E)}$.

Let $L_{\text {loc }}^{1}\left(\mathbb{R}^{n}\right)$ be the collection of all locally integrable functions on $\mathbb{R}^{n}$. Given a function $f \in L_{\text {loc }}^{1}\left(\mathbb{R}^{n}\right)$, the HardyLittlewood maximal operator $\mathscr{M}$ is defined by

$$
\mathscr{M} f(x):=\sup _{r>0} r^{-n} \int_{B(x, r)}|f(y)| \mathrm{d} y, \quad \forall x \in \mathbb{R}^{n},
$$

where and what follows $B(x, r):=\left\{y \in \mathbb{R}^{n}:|x-y|<r\right\}$. We also use the following notation: $p_{-}:=\operatorname{ess} \inf \left\{p(x): x \in \mathbb{R}^{n}\right\}$ and $p_{+}:=\operatorname{ess} \sup \left\{p(x): x \in \mathbb{R}^{n}\right\}$. The set $\mathscr{P}\left(\mathbb{R}^{n}\right)$ consists of all $p(\cdot)$ satisfying $p_{-}>1$ and $p_{+}<\infty . \mathscr{B}\left(\mathbb{R}^{n}\right)$ is the set of $p(\cdot) \in \mathscr{P}\left(\mathbb{R}^{n}\right)$ satisfying the condition that $\mathscr{M}$ is bounded on $L^{p(\cdot)}\left(\mathbb{R}^{n}\right)$. It is well known that if $p(\cdot) \in \mathscr{P}\left(\mathbb{R}^{n}\right)$ satisfies the following global log-Hölder continuous then $p(\cdot) \in \mathscr{B}\left(\mathbb{R}^{n}\right)$; see [37-42].

Definition 2. Let $\alpha(\cdot)$ be a real-valued function on $\mathbb{R}^{n}$. If there exists $C>0$ such that, for all $x, y \in \mathbb{R}^{n},|x-y|<1 / 2$,

$$
|\alpha(x)-\alpha(y)| \leqslant \frac{C}{-\log (|x-y|)},
$$

then $\alpha(\cdot)$ is said local log-Hölder continuous on $\mathbb{R}^{n}$.

If there exists $C>0$, such that for all $x \in \mathbb{R}^{n}$,

$$
|\alpha(x)-\alpha(0)| \leqslant \frac{C}{\log (e+1 /|x|)},
$$

then $\alpha(\cdot)$ is said log-Hölder continuous at origin.
If there exist $\alpha_{\infty} \in \mathbb{R}$ and a constant $C>0$ such that for all $x \in \mathbb{R}^{n}$

$$
\left|\alpha(x)-\alpha_{\infty}\right| \leqslant \frac{C}{\log (e+|x|)}
$$

then $\alpha(\cdot)$ is said log-Hölder continuous at infinity.

If $\alpha(\cdot)$ is both local log-Hölder continuous and log-Hölder continuous at infinity, then $\alpha(\cdot)$ is said global log-Hölder continuous.

The sets of log-Hölder continuous functions, log-Hölder continuous functions at origin, log-Hölder continuous functions at infinity, global log-Hölder continuous are denoted by $\mathscr{P}_{\operatorname{loc}}^{\log }\left(\mathbb{R}^{n}\right), \mathscr{P}_{0}^{\log }\left(\mathbb{R}^{n}\right), \mathscr{P}_{\infty}^{\log }\left(\mathbb{R}^{n}\right)$, and $\mathscr{P}^{\log }\left(\mathbb{R}^{n}\right)$, respectively.

We denote $p^{\prime}(\cdot)$ by the conjugate exponent to $p(\cdot)$, which means $p^{\prime}(\cdot)=p(\cdot) /(p(\cdot)-1)$. It is also well known that $p(\cdot) \in$ $\mathscr{B}\left(\mathbb{R}^{n}\right)$ is equivalent to $p^{\prime}(\cdot) \in \mathscr{B}\left(\mathbb{R}^{n}\right)$; see [39].

For simplicity, we denote $L^{p(\cdot)}\left(\mathbb{R}^{n}\right)$ by $L^{p(\cdot)}$. We will use the following results.

Lemma 3 (see [43]). Let $p(\cdot) \in \mathscr{P}\left(\mathbb{R}^{n}\right)$. If $f \in L^{p(\cdot)}$ and $g \in$ $L^{p^{\prime}(\cdot)}$, then $f g$ is integrable on $\mathbb{R}^{n}$ and

$$
\int_{\mathbb{R}^{n}}|f(x) g(x)| d x \leqslant r_{p}\|f\|_{L^{p(\cdot)}}\|g\|_{L^{p^{\prime}(\cdot)}}
$$

where $r_{p}=1+1 / p^{-}-1 / p^{+}$.

Lemma 4 (see [27]). Let $p(\cdot) \in \mathscr{B}\left(\mathbb{R}^{n}\right)$. Then there exist $0<$ $\delta_{1}, \delta_{2}<1$, and a positive constant $C$ depending only on $p(\cdot)$ and $n$ such that for all balls $B$ in $\mathbb{R}^{n}$ and all measurable subsets $S \subset B$,

$$
\begin{aligned}
\frac{\left\|\chi_{B}\right\|_{L^{p(\cdot)}}}{\left\|\chi_{S}\right\|_{L^{p(\cdot)}}} \leqslant & C \frac{|B|}{|S|}, \quad \frac{\left\|\chi_{S}\right\|_{L^{p(\cdot)}}}{\left\|\chi_{B}\right\|_{L^{p(\cdot)}}} \leqslant C\left(\frac{|S|}{|B|}\right)^{\delta_{1}}, \\
& \frac{\left\|\chi_{S}\right\|_{L^{p^{\prime}(\cdot)}}}{\left\|\chi_{B}\right\|_{L^{p^{\prime}(\cdot)}}} \leqslant C\left(\frac{|S|}{|B|}\right)^{\delta_{2}} .
\end{aligned}
$$

Lemma 5 (see [27]). Let $p(\cdot) \in \mathscr{B}\left(\mathbb{R}^{n}\right)$. Then there exists a positive constant $C$ such that, for any ball $B$ in $\mathbb{R}^{n}$,

$$
\left\|\chi_{B}\right\|_{L^{p(\cdot)}}\left\|\chi_{B}\right\|_{L^{p^{\prime}(\cdot)}} \leqslant C|B|
$$

To give the definition of Herz-Morrey spaces with variable exponents, let us introduce the following notations. Let $k \in \mathbb{Z}, B_{k}:=\left\{x \in \mathbb{R}^{n}:|x| \leqslant 2^{k}\right\}, L \in \mathbb{Z}, D_{k}:=B_{k} \backslash B_{k-1}$, and $\chi_{k}:=\chi_{D_{k}}$. The symbol $\mathbb{N}_{0}$ denotes the set of all nonnegative integers. For $m \in \mathbb{N}_{0}$, we denote $\widetilde{\chi}_{m}:=\chi_{D_{m}}$ if $m \geq 1$ and $\tilde{\chi}_{0}:=\chi_{B_{0}}$.

Definition 6. Let $0<q \leqslant \infty, p(\cdot) \in \mathscr{P}\left(\mathbb{R}^{n}\right)$, and $0 \leq \lambda<$ $\infty$. Let $\alpha(\cdot)$ be a bounded real-valued measurable function on $\mathbb{R}^{n}$. The homogeneous Herz-Morrey space $M \dot{K}_{p(\cdot), \lambda}^{\alpha(\cdot), q}$ and 
nonhomogeneous Herz-Morrey space $M K_{p(\cdot), \lambda}^{\alpha(\cdot), q}$ are defined, respectively, by

$$
\begin{aligned}
M \dot{K}_{p(\cdot), \lambda}^{\alpha(\cdot), q} & :=\left\{f \in L_{\text {loc }}^{p(\cdot)}\left(\mathbb{R}^{n} \backslash 0\right):\|f\|_{M \dot{K}_{p(\cdot), \lambda}^{\alpha(\cdot), q}}<\infty\right\}, \\
M K_{p(\cdot), \lambda}^{\alpha(\cdot), q} & :=\left\{f \in L_{\text {loc }}^{p(\cdot)}\left(\mathbb{R}^{n}\right):\|f\|_{\left.M K_{p(\cdot), \lambda}^{\alpha(\cdot),}<\infty\right\},}<\right.
\end{aligned}
$$

where

$$
\begin{aligned}
& \|f\|_{M \dot{K}_{p(\cdot), \lambda}^{\alpha(\cdot), q}}:=\sup _{L \in \mathbb{Z}} 2^{-L \lambda}\left(\sum_{k=-\infty}^{L}\left\|2^{\alpha(\cdot) k} f \chi_{k}\right\|_{L^{p(\cdot)}}^{q}\right)^{1 / q}, \\
& \|f\|_{M K_{p(\cdot), \lambda}^{\alpha(\cdot), q}}:=\sup _{L \in \mathbb{N}_{0}} 2^{-L \lambda}\left(\sum_{k=0}^{L}\left\|2^{\alpha(\cdot) k} f \tilde{\chi}_{k}\right\|_{L^{p(\cdot)}}^{q}\right)^{1 / q} .
\end{aligned}
$$

Here there is the usual modification when $q=\infty$.

Proposition 7. Let $p(\cdot) \in \mathscr{P}\left(\mathbb{R}^{n}\right), q \in(0, \infty]$, and $\lambda \in$ $[0, \infty)$. If $\alpha(\cdot) \in L^{\infty}\left(\mathbb{R}^{n}\right) \cap \mathscr{P}_{0}^{\log }\left(\mathbb{R}^{n}\right) \cap \mathscr{P}_{\infty}^{\log }\left(\mathbb{R}^{n}\right)$, then

$$
\begin{array}{r}
\|f\|_{M \dot{K}_{p(\cdot), \lambda}^{\alpha(\cdot), q}} \\
\approx \max \left\{\sup _{L \leqslant 0, L \in \mathbb{Z}} 2^{-L \lambda}\left(\sum_{k=-\infty}^{L} 2^{k \alpha(0) q}\left\|f \chi_{k}\right\|_{L^{p(\cdot)}}^{q}\right)^{1 / q},\right. \\
\sup _{L>0, L \in \mathbb{Z}}\left[2^{-L \lambda}\left(\sum_{k=-\infty}^{-1} 2^{k \alpha(0) q}\left\|f \chi_{k}\right\|_{L^{p(\cdot)}}^{q}\right)^{1 / q}\right. \\
\left.\left.+2^{-L \lambda}\left(\sum_{k=0}^{L} 2^{k \alpha(\infty) q}\left\|f \chi_{k}\right\|_{L^{p(\cdot)}}^{q}\right)^{1 / q}\right]\right\} .
\end{array}
$$

Proposition 7 is the generalization of Herz spaces with variable exponents in [28], and it was used in [33, 34].

Lemma 8. Let $p(\cdot) \in \mathscr{B}\left(\mathbb{R}^{n}\right), 0<q<\infty$, and $\lambda \in[0, \infty)$. Let $\alpha(\cdot)$ be bounded and log-Hölder continuous both at the origin and at infinity such that $-n \delta_{1}<\alpha(0) \leqslant \alpha_{\infty}<n \delta_{2}$, where $0<\delta_{1}, \delta_{2}<1$ are constants in Lemma 4. Suppose that $T$ is a sublinear and bounded operator on $L^{p(\cdot)}$ satisfying size condition

$$
|T f(x)| \leqslant C \int_{\mathbb{R}^{n}}|x-y|^{-n}|f(y)| d y
$$

for all $f \in L_{\text {loc }}^{1}\left(\mathbb{R}^{n}\right)$ with compact support and a.e. $x \notin \operatorname{supp} f$. Then there exists a positive constant $C$ such that

$$
\|T f\|_{M \dot{K}_{p(\cdot), \lambda}^{\alpha(\cdot), q}} \leqslant C\|f\|_{M \dot{K}_{p(\cdot), \lambda}^{\alpha(\cdot), q},}, \quad\|T f\|_{M K_{p(\cdot), \lambda}^{\alpha(\cdot), q}} \leqslant C\|f\|_{M K_{p(\cdot), \lambda}^{\alpha(\cdot), q}}
$$

for any function $f$ belongs to $M \dot{K}_{p(\cdot), \lambda}^{\alpha(\cdot), q}$ and $M K_{p(\cdot), \lambda}^{\alpha(\cdot), q}$, respectively.

Lemma 8 is the generalization of Herz spaces with variable exponents in [28]. For a proof, see [33].

\section{The Atomic Characterization}

In this section, we will introduce Herz-Morrey-Hardy spaces with variable exponents $H M \dot{K}_{p(\cdot), \lambda}^{\alpha(\cdot), q}$ and $H M K_{p(\cdot), \lambda}^{\alpha(\cdot), q}$. To do this, we need to recall some notations. $\mathcal{S}\left(\mathbb{R}^{n}\right)$ denotes the Schwartz space of all rapidly decreasing infinitely differentiable functions on $\mathbb{R}^{n}$, and $\mathcal{S}^{\prime}\left(\mathbb{R}^{n}\right)$ denotes the dual space of $\mathcal{S}\left(\mathbb{R}^{n}\right)$. Let $G_{N} f$ be the grand maximal function of $f$ defined by

$$
G_{N} f(x):=\sup _{\phi \in \mathscr{A}_{N}}\left|\phi_{\nabla}^{*}(f)(x)\right|, \quad x \in \mathbb{R}^{n}
$$

where $\mathscr{A}_{N}:=\left\{\phi \in \mathcal{S}\left(\mathbb{R}^{n}\right): \sup _{|\alpha|,|\beta| \leqslant N, \forall x \in \mathbb{R}^{n}}\left|x^{\alpha} D^{\beta} \phi(x)\right| \leqslant 1\right\}$ and $N>n+1$ and $\phi_{\nabla}^{*}$ is the nontangential maximal operator defined by

$$
\begin{gathered}
\phi_{\nabla}^{*}(f)(x):=\sup _{|y-x|<t}\left|\phi_{t} * f(y)\right|, \\
\forall x \in \mathbb{R}^{n} \quad \text { with } \phi_{t}(\cdot)=t^{-n} \phi\left(\begin{array}{l}
\cdot \\
t
\end{array}\right) .
\end{gathered}
$$

The grand maximal operator $G_{N}$ was firstly introduced by Fefferman and Stein in [44] to study classical Hardy spaces. For classical Hardy spaces, one can also see [45-47]. Nakai and Sawano generalized them to variable exponent case in [22].

Definition 9. Let $\alpha(\cdot) \in L^{\infty}\left(\mathbb{R}^{n}\right), 0<q \leq \infty, p(\cdot) \in \mathscr{P}\left(\mathbb{R}^{n}\right)$, $0 \leq \lambda<\infty$, and $N>n+1$. The homogeneous HerzMorrey-Hardy space with variable exponents $H M \dot{K}_{p(\cdot), \lambda}^{\alpha(\cdot),}$ and nonhomogeneous Herz-Morrey-Hardy space with variable exponents $H M K_{p(\cdot), \lambda}^{\alpha(\cdot),}$ are defined, respectively, by

$$
\begin{aligned}
& H M \dot{K}_{p(\cdot), \lambda}^{\alpha(\cdot), q} \\
& \quad:=\left\{f \in \mathcal{S}^{\prime}\left(\mathbb{R}^{n}\right):\|f\|_{H M \dot{K}_{p(\cdot), \lambda}^{\alpha(\cdot), q}}:=\left\|G_{N} f\right\|_{M \dot{K}_{p(\cdot), \lambda}^{\alpha(\cdot), q}}<\infty\right\}, \\
& H M K_{p(\cdot), \lambda}^{\alpha(\cdot), q} \\
& \quad:=\left\{f \in \mathcal{S}^{\prime}\left(\mathbb{R}^{n}\right):\|f\|_{H M K_{p(\cdot), \lambda}^{\alpha(\cdot), q}}:=\left\|G_{N} f\right\|_{M K_{p(\cdot), \lambda}^{\alpha(\cdot), q}}<\infty\right\} .
\end{aligned}
$$

Remark 10. If $\alpha(\cdot) \equiv \alpha$ and $\lambda=0$, these spaces were considered by Wang and Liu in [31]. If $p(\cdot)$ and $\alpha(\cdot)$ are constant and $\lambda=0$, these are the classical Herz type Hardy spaces; see [6].

Let $\psi(r)=1$ for $r \in[0,1]$ and $\psi(r)=r^{-N}$ for $r \in(1,+\infty)$. Then there exists $C>0$ such that $\phi(x) \leq C \psi(|x|)$ for all $\phi \epsilon$ $\mathscr{A}_{N}$. Therefore, by [46, Proposition in Page 57], there exists $C>0$ such that $G_{N} f(x) \leqslant C M f(x)$ for all $x \in \mathbb{R}^{n}$. This means that $G_{N} f$ satisfies the size condition in Lemma 8. By Lemma 8 , if $-n \delta_{1}<\alpha(0) \leqslant \alpha_{\infty}<n \delta_{2}$ and $p(\cdot) \in \mathscr{B}\left(\mathbb{R}^{n}\right)$, then

$$
\begin{gathered}
H M \dot{K}_{p(\cdot), \lambda}^{\alpha(\cdot), q} \cap L_{\text {loc }}^{p(\cdot)}\left(\mathbb{R}^{n} \backslash\{0\}\right)=M \dot{K}_{p(\cdot, \lambda)}^{\alpha(\cdot), q}, \\
H M K_{p(\cdot), \lambda}^{\alpha(\cdot), q} \cap L_{\text {loc }}^{p(\cdot)}\left(\mathbb{R}^{n}\right)=M K_{p(\cdot), \lambda}^{\alpha(\cdot), q} .
\end{gathered}
$$


Thus we are interested in the case $n \delta_{2} \leqslant \alpha(0), \alpha_{\infty}<\infty$. In this case, we will establish a characterization of the spaces $H M \dot{K}_{p(\cdot), \lambda}^{\alpha(\cdot), q}$ and $H M K_{p(\cdot), \lambda}^{\alpha(\cdot), q}$ in terms of central atom. For $u \in \mathbb{R}$ we denote by $[u]$ the largest integer less than or equal to $u$.

Definition 11. Let $p(\cdot) \in \mathscr{P}\left(\mathbb{R}^{n}\right)$ and $\alpha(\cdot) \in L^{\infty}\left(\mathbb{R}^{n}\right)$ be log-Hölder continuous both at the origin and infinity, and nonnegative integer $s \geqslant\left[\alpha_{r}-n \delta_{2}\right]$; here $\alpha_{r}=\alpha(0)$, if $r<1$, and $\alpha_{r}=\alpha_{\infty}$, if $r \geqslant 1, n \delta_{2} \leqslant \alpha_{r}<\infty$ and $\delta_{2}$ as in Lemma 4 .

(i) A function $a$ on $\mathbb{R}^{n}$ is called a central $(\alpha(\cdot), p(\cdot))$ atom, if it satisfies (1) supp $a \subset B(0, r) ;(2)\|a\|_{L^{p(\cdot)}} \leqslant$ $|B(0, r)|^{-\alpha_{r} / n} ;(3) \int_{\mathbb{R}^{n}} a(x) x^{\beta} \mathrm{d} x=0,|\beta| \leqslant s$.

(ii) A function $a$ on $\mathbb{R}^{n}$ is called a central $(\alpha(\cdot), p(\cdot))$-atom of restricted type, if it satisfies (1) supp $a \subset B(0, r)$, $r \geqslant 1 ;(2)\|a\|_{L^{p(\cdot)}} \leqslant|B(0, r)|^{-\alpha_{r} / n} ;(3) \int_{\mathbb{R}^{n}} a(x) x^{\beta} \mathrm{d} x=$ $0,|\beta| \leqslant s$.

Remark 12. If $p(\cdot) \equiv p$ and $\alpha(\cdot) \equiv \alpha$ are constant, then taking $\delta_{2}=1-1 / p$ we recover the classical case in [6].

Theorem 13. Let $0<q<\infty, p(\cdot) \in \mathscr{B}\left(\mathbb{R}^{n}\right), 0 \leq \lambda<\infty$, and $\alpha(\cdot) \in L^{\infty}\left(\mathbb{R}^{n}\right)$ be log-Hölder continuous both at the origin and infinity, $2 \lambda \leqslant \alpha(\cdot), n \delta_{2} \leqslant \alpha(0), \alpha_{\infty}<\infty$, and $\delta_{2}$ as in Lemma 4.

(i) $f \in H M \dot{K}_{p(\cdot), \lambda}^{\alpha(\cdot), q}$ if and only if $f=\sum_{k=-\infty}^{\infty} \lambda_{k} a_{k}$ in the sense of $\delta^{\prime}\left(\mathbb{R}^{n}\right)$, where each $a_{k}$ is a central $(\alpha(\cdot), p(\cdot))$-atom with support contained in $B_{k}$ and $\sup _{L \in \mathbb{Z}} 2^{-L \lambda} \sum_{k=-\infty}^{L}\left|\lambda_{k}\right|^{q}<\infty$. Moreover,

$$
\|f\|_{H M \dot{K}_{p(\cdot), \lambda}^{\alpha(\cdot), q}} \approx \inf \sup _{L \in \mathbb{Z}} 2^{-L \lambda}\left(\sum_{k=-\infty}^{L}\left|\lambda_{k}\right|^{q}\right)^{1 / q},
$$

where the infimum is taken over all above decompositions of $f$.

(ii) $f \in H M K_{p(\cdot), \lambda}^{\alpha(\cdot), q}$ if and only if $f=\sum_{k=0}^{\infty} \lambda_{k} a_{k}$ in the sense of $\delta^{\prime}\left(\mathbb{R}^{n}\right)$, where each $a_{k}$ is a central $(\alpha(\cdot), p(\cdot))$ atom of restricted type with support contained in $B_{k}$ and $\sup _{L \in \mathbb{Z}} 2^{-L \lambda} \sum_{k=0}^{L}\left|\lambda_{k}\right|^{q}<\infty$. Moreover

$$
\|f\|_{H M K_{p(\cdot), \lambda}^{\alpha(\cdot), q}} \approx \inf \sup _{L \in \mathbb{Z}} 2^{-L \lambda}\left(\sum_{k=0}^{L}\left|\lambda_{k}\right|^{q}\right)^{1 / q},
$$

where the infimum is taken over all above decompositions of $f$.

Proof. We only prove (i). The proof of (ii) is similar. We use the ideas in [6]. To prove the necessity, we choose $\phi \in$ $C_{0}^{\infty}\left(\mathbb{R}^{n}\right)$ such that $\phi \geqslant 0, \int_{\mathbb{R}^{n}} \phi(x) \mathrm{d} x=1$, and supp $\phi \subset\{x$ : $|x| \leqslant 1\}$. For $j \in \mathbb{N}_{0}$, let $\phi_{(j)}(x):=2^{j n} \phi\left(2^{j} x\right), \forall x \in \mathbb{R}^{n}$. For each $f \in \mathcal{S}^{\prime}\left(\mathbb{R}^{n}\right)$, set $f^{(j)}(x)=f * \phi_{(j)}(x), \forall x \in \mathbb{R}^{n}$. It is obvious that $f^{(j)} \in C^{\infty}\left(\mathbb{R}^{n}\right)$ and $\lim _{j \rightarrow \infty} f^{(j)}=f$ in $\mathcal{S}^{\prime}\left(\mathbb{R}^{n}\right)$. Let $\psi$ be a radial smooth function such that supp $\psi \subset\{x$ :
$1 / 2-\varepsilon \leqslant|x| \leqslant 1+\varepsilon\}$ with $0<\varepsilon<1 / 4, \psi(x)=1$ for $1 / 2 \leqslant|x| \leqslant 1$. Let $\psi_{k}(x):=\psi\left(2^{-k} x\right)$ for $k \in \mathbb{Z}$ and

$$
\widetilde{A}_{k, \varepsilon}:=\left\{x: 2^{k-1}-2^{k} \varepsilon \leqslant|x| \leqslant 2^{k}+2^{k} \varepsilon\right\} .
$$

Observe that supp $\psi_{k} \subset \widetilde{A}_{k, \varepsilon}$ and $\psi_{k}(x)=1$ for $x \in A_{k}:=$ $\left\{x: 2^{k-1} \leqslant|x| \leqslant 2^{k}\right\}$. Obviously, $1 \leqslant \sum_{k=-\infty}^{\infty} \psi_{k}(x) \leqslant 2, x \neq 0$. Let

$$
\Phi_{k}(x):= \begin{cases}\frac{\psi_{k}(x)}{\sum_{l=-\infty}^{\infty} \psi_{l}(x)}, & x \neq 0 \\ 0, & x=0\end{cases}
$$

Then $\sum_{k=-\infty}^{\infty} \Phi_{k}(x)=1$ for $x \neq 0$. For each $m \in \mathbb{N}$, we denote by $\mathscr{P}_{m}$ the class of all the real polynomials with the degree less than $m$. Let $P_{k}^{(j)}(x):=P_{\widetilde{A}_{k, \varepsilon}}\left(f^{(j)} \Phi_{k}\right)(x) \chi_{\widetilde{A}_{k, \varepsilon}} \in \mathscr{P}_{m}\left(\mathbb{R}^{n}\right)$ be the unique polynomial satisfying

$$
\begin{gathered}
\int_{\widetilde{A}_{k, \varepsilon}}\left(f^{(j)}(x) \Phi_{k}(x)-P_{k}^{(j)}(x)\right) x^{\beta} \mathrm{d} x=0, \\
|\beta| \leqslant m=\max \left\{\left[\alpha(0)-n \delta_{2}\right],\left[\alpha_{\infty}-n \delta_{2}\right]\right\} .
\end{gathered}
$$

Write

$$
\begin{aligned}
f^{(j)}(x) & =\sum_{k=-\infty}^{\infty}\left(f^{(j)}(x) \Phi_{k}(x)-P_{k}^{(j)}(x)\right)+\sum_{k=-\infty}^{\infty} P_{k}^{(j)}(x) \\
& :=I_{(j)}+I I_{(j)} .
\end{aligned}
$$

For the term $I_{(j)}$, let $g_{k}^{(j)}(x):=f^{(j)}(x) \Phi_{k}(x)-$ $P_{k}^{(j)}(x)$ and $a_{k}^{(j)}(x) \quad:=g_{k}^{(j)}(x) / \lambda_{k}$, where $\lambda_{k}:=$ $b\left|B_{k+1}\right|^{\alpha_{k+1} / n} \sum_{l=k-1}^{k+1}\left\|\left(G_{N} f\right) \chi_{l}\right\|_{L^{p(\cdot)}}$ and $b$ is a constant which will be chosen later. Note that supp $a_{k}^{(j)} \subset B_{k+1}, I_{(j)}=$ $\sum_{k=-\infty}^{\infty} \lambda_{k} a_{k}^{(j)}(x)$.

Now we estimate $\left\|g_{k}^{(j)}\right\|_{L^{p(\cdot)}}$. To do this, let $\left\{\phi_{\gamma}^{k}:|\gamma| \leqslant m\right\}$ be the orthogonal polynomials restricted to $\widetilde{A}_{k, \varepsilon}$ with respect to the weight $1 /\left|\widetilde{A}_{k, \varepsilon}\right|$, which are obtained from $\left\{x^{\beta}:|\beta| \leqslant m\right\}$ by the Gram-Schmidt method, which means

$$
\left\langle\phi_{\nu}^{k}, \phi_{\mu}^{k}\right\rangle=\frac{1}{\left|\widetilde{A}_{k, \varepsilon}\right|} \int_{\widetilde{A}_{k, \varepsilon}} \phi_{\nu}^{k}(x) \phi_{\mu}^{k}(x) \mathrm{d} x=\delta_{\nu \mu},
$$

where $\delta_{\nu \mu}=1$ for $\nu=\mu$, otherwise 0 .

It is easy to see that $P_{k}^{(j)}(x)=\sum_{|\gamma| \leqslant m}\left\langle f^{(j)} \Phi_{k}, \phi_{\gamma}^{k}\right\rangle \phi_{\gamma}^{k}(x)$ for $x \in \widetilde{A}_{k, \varepsilon}$. On the other hand, from $\left(1 /\left|\widetilde{A}_{k, \varepsilon}\right|\right) \int_{\widetilde{A}_{k, \varepsilon}} \phi_{\nu}^{k}(x) \phi_{\mu}^{k}(x) \mathrm{d} x=\delta_{\nu \mu}$ we infer that

$$
\frac{1}{\left|\widetilde{A}_{1, \varepsilon}\right|} \int_{\widetilde{A}_{1, \varepsilon}} \phi_{\nu}^{k}\left(2^{k-1} y\right) \phi_{\mu}^{k}\left(2^{k-1} y\right) \mathrm{d} y=\delta_{\nu \mu} .
$$

Thus, we deduce $\phi_{\nu}^{k}\left(2^{k-1} y\right)=\phi_{\nu}^{1}(y)$ a.e. That is, $\phi_{\nu}^{k}(x)=$ $\phi_{\nu}^{1}\left(2^{1-k} x\right)$ almost everywhere for $x \in \widetilde{A}_{k, \varepsilon}$. Therefore 
Journal of Function Spaces

5

$\left|\phi_{\nu}^{k}(x)\right| \leqslant C$ for $x \in \widetilde{A}_{k, \varepsilon}$. By the generalized Hölder inequality we have

$$
\begin{aligned}
\left|P_{k}^{(j)}(x)\right| & \lesssim \frac{1}{\left|\widetilde{A}_{k, \varepsilon}\right|} \int_{\widetilde{A}_{k, \varepsilon}}\left|f^{(j)}(x) \Phi_{k}(x)\right| \mathrm{d} x \\
& \lesssim \frac{1}{\left|\widetilde{A}_{k, \varepsilon}\right|}\left\|f^{(j)} \Phi_{k}\right\|_{L^{p(\cdot)}}\left\|\chi_{\widetilde{A}_{k, \varepsilon}}\right\|_{L^{p^{\prime}(\cdot)}} .
\end{aligned}
$$

By Lemma 5 we have

$$
\begin{aligned}
&\left\|g_{k}^{(j)}\right\|_{L^{p(\cdot)}} \leqslant\left\|f^{(j)} \Phi_{k}\right\|_{L^{p(\cdot)}}+\left\|P_{k}^{(j)}\right\|_{L^{p(\cdot)}} \\
& \leq\left\|f^{(j)} \Phi_{k}\right\|_{L^{p(\cdot)}}+\frac{1}{\left|\widetilde{A}_{k, \varepsilon}\right|}\left\|f^{(j)} \Phi_{k}\right\|_{L^{p(\cdot)}} \\
& \times\left\|\chi_{\widetilde{A}_{k, \varepsilon}}\right\|_{L^{p^{\prime}(\cdot)}}\left\|\chi_{\widetilde{A}_{k, \varepsilon}}\right\|_{L^{p(\cdot)}} \\
& \leq\left\|f^{(j)} \Phi_{k}\right\|_{L^{p(\cdot)}}+\left\|f^{(j)} \Phi_{k}\right\|_{L^{p(\cdot)}} \\
& \leq\left\|\left(f * \phi_{(j)}\right) \Phi_{k}\right\|_{L^{p(\cdot)}} \\
& \leqslant C \sum_{l=k-1}^{k+1}\left\|\left(G_{N} f\right) \chi_{l}\right\|_{L^{p(\cdot)}} .
\end{aligned}
$$

Choose $b=C$; then $\left\|a_{k}^{(j)}\right\|_{L^{p(\cdot)}} \leqslant\left|B_{k+1}\right|^{-\alpha_{k+1} / n}$ and each $a_{k}^{(j)}$ is a central $(\alpha(\cdot), p(\cdot))$-atom with support contained in $B_{k+1}$. Here and below we abuse $\alpha_{k}:=\alpha_{2^{k}}$ and it is well defined in Definition 11. Thus,

$$
\begin{aligned}
& \sup _{L \in \mathbb{Z}} 2^{-L \lambda q} \sum_{k=-\infty}^{L}\left|\lambda_{k}\right|^{q} \\
& \quad \leq \sup _{L \in \mathbb{Z}} 2^{-L \lambda q} \sum_{k=-\infty}^{L}\left|B_{k+1}\right|^{q \alpha_{k+1} / n}\left(\sum_{l=k-1}^{k+1}\left\|\left(G_{N} f\right) \chi_{l}\right\|_{L^{p(\cdot)}}\right)^{q} \\
& \quad \leq \sup _{L \in \mathbb{Z}} 2^{-L \lambda q} \sum_{k=-\infty}^{L}\left|B_{k+1}\right|^{q \alpha_{k+1} / n}\left\|\left(G_{N} f\right) \chi_{k}\right\|_{L^{p \cdot(\cdot)}}^{q} \\
& :=A .
\end{aligned}
$$

Now we estimate $A$. By the condition of $\alpha(\cdot)$ and Proposition 7 we consider it in two cases.

Case $1(L \leqslant 0)$. Consider

$$
\begin{aligned}
2^{-L \lambda q} & \sum_{k=-\infty}^{L}\left|B_{k+1}\right|^{q \alpha_{k+1} / n}\left\|\left(G_{N} f\right) \chi_{k}\right\|_{L^{p(\cdot)}}^{q} \\
& \leq \sup _{L \leqslant 0, L \in \mathbb{Z}} 2^{-L \lambda q} \sum_{k=-\infty}^{L} 2^{k q \alpha(0)}\left\|\left(G_{N} f\right) \chi_{k}\right\|_{L^{p(\cdot)}}^{q} \\
& \leq\left\|G_{N} f\right\|_{M K_{p(\cdot), \lambda}^{\alpha(\cdot), q}}^{q}
\end{aligned}
$$

Case $2(L>0)$. Consider

$$
\begin{aligned}
2^{-L \lambda q} & \sum_{k=-\infty}^{L}\left|B_{k+1}\right|^{q \alpha_{k+1} / n}\left\|\left(G_{N} f\right) \chi_{k}\right\|_{L^{p(\cdot)}}^{q} \\
& =2^{-L \lambda q} \sum_{k=-\infty}^{-2} 2^{(k+1) q \alpha(0)}\left\|\left(G_{N} f\right) \chi_{k}\right\|_{L^{p(\cdot)}}^{q}
\end{aligned}
$$

$$
\begin{gathered}
+2^{-L \lambda q} \sum_{k=-1}^{L} 2^{(k+1) q \alpha(\infty)}\left\|\left(G_{N} f\right) \chi_{k}\right\|_{L^{p(\cdot)}}^{q} \\
\leq 2^{-L \lambda q} \sum_{k=-\infty}^{-1} 2^{k q \alpha(0)}\left\|\left(G_{N} f\right) \chi_{k}\right\|_{L^{p(\cdot)}}^{q} \\
+2^{-L \lambda q} \sum_{k=0}^{L} 2^{k q \alpha(\infty)}\left\|\left(G_{N} f\right) \chi_{k}\right\|_{L^{p(\cdot)}}^{q} \\
\leq\left\|G_{N} f\right\|_{M K_{p(\cdot), \lambda}^{\alpha(\cdot),}}^{q} \cdot \\
\sup _{L \in \mathbb{Z}} 2^{-L \lambda q} \sum_{k=-\infty}^{L}\left|\lambda_{k}\right|^{q} \lesssim\left\|G_{N} f\right\|_{M K_{p(\cdot), \lambda}^{\alpha(\cdot),}}^{p} .
\end{gathered}
$$

Hence,

It remains to estimate $I I_{(j)}$. Let $\left\{\psi_{d}^{k}:|\gamma| \leqslant m\right\}$ be the dual basis of $\left\{x^{\beta}:|\beta| \leqslant m\right\}$ with respect to the weight $1 /\left|\widetilde{A}_{k, \varepsilon}\right|$ on $\widetilde{A}_{k, \varepsilon}$, that is,

$$
\left\langle\psi_{\gamma}^{k}, x^{\beta}\right\rangle=\frac{1}{\left|\widetilde{A}_{k, \varepsilon}\right|} \int_{\widetilde{A}_{k, \varepsilon}} x^{\beta} \psi_{\gamma}^{k}(x) \mathrm{d} x=\delta_{\beta \gamma} .
$$

Similar to the method of [48], let

$$
\begin{aligned}
h_{k, \gamma}^{(j)}(x):= & \sum_{l=-\infty}^{k}\left(\frac{\psi_{\gamma}^{k}(x) \chi_{\widetilde{A}_{k, \varepsilon}}(x)}{\left|\widetilde{A}_{k, \varepsilon}\right|}-\frac{\psi_{\gamma}^{k+1}(x) \chi_{\widetilde{A}_{k+1, \varepsilon}}(x)}{\left|\widetilde{A}_{k+1, \varepsilon}\right|}\right) \\
& \times \int_{\mathbb{R}^{n}} f^{(j)}(y) \Phi_{l}(y) y^{\gamma} \mathrm{d} y .
\end{aligned}
$$

We write

$$
\begin{aligned}
I I_{(j)}= & \sum_{k=-\infty}^{\infty} \sum_{|\gamma| \leqslant m}\left\langle f^{(j)} \Phi_{k}, x^{\gamma}\right\rangle \psi_{\gamma}^{k}(x) \chi_{\widetilde{A}_{k, \varepsilon}}(x) \\
= & \sum_{|\gamma| \leqslant m} \sum_{k=-\infty}^{\infty}\left(\int_{\mathbb{R}^{n}} f^{(j)} \Phi_{k} x^{\gamma} \mathrm{d} x\right) \frac{\psi_{\gamma}^{k}(x) \chi_{\widetilde{A}_{k, \varepsilon}}(x)}{\left|\widetilde{A}_{k, \varepsilon}\right|} \\
= & \sum_{|\gamma| \leqslant m} \sum_{k=-\infty}^{\infty}\left(\sum_{l=-\infty}^{k} \int_{\mathbb{R}^{n}} f^{(j)}(x) \Phi_{l}(x) x^{\gamma} \mathrm{d} x\right) \\
& \times\left(\frac{\psi_{\gamma}^{k}(x) \chi_{\widetilde{A}_{k, \varepsilon}}(x)}{\left|\widetilde{A}_{k, \varepsilon}\right|}-\frac{\psi_{\gamma}^{k+1}(x) \chi_{\widetilde{A}_{k+1, \varepsilon}}(x)}{\left|\widetilde{A}_{k+1, \varepsilon}\right|}\right) \\
= & : \sum_{|\gamma| \leqslant m} \sum_{k=-\infty}^{\infty} \frac{\alpha_{k, \gamma} h_{k, \gamma}^{(j)}(x)}{\alpha_{k, \gamma}} \\
= & : \sum_{|\gamma| \leqslant m} \sum_{k=-\infty}^{\infty} \alpha_{k, \gamma} a_{k, \gamma}^{(j)}(x),
\end{aligned}
$$

where

$$
\alpha_{k, \gamma}:=\widetilde{b} \sum_{l=k-1}^{k+1}\left\|\left(G_{N} f\right) \chi_{l}\right\|_{L^{p(\cdot)}}\left|B_{k+2}\right|^{\alpha_{k+2} / n}
$$


and $\widetilde{b}$ is a constant which will be chosen later. Note that

$$
\begin{aligned}
\int_{\mathbb{R}^{n}} \sum_{l=-\infty}^{k}\left|\Phi_{l}(x) x^{\gamma}\right| \mathrm{d} x & =\sum_{l=-\infty}^{k} \int_{\widetilde{A}_{k, \varepsilon}}\left|\Phi_{l}(x) x^{\gamma}\right| \mathrm{d} x \\
& \lesssim 2^{k(n+|\gamma|)} .
\end{aligned}
$$

By a computation we have

$$
\begin{array}{r}
\left|\int_{\mathbb{R}^{n}} f^{(j)}(y) \sum_{l=-\infty}^{k} \Phi_{l}(y) y^{\gamma} \mathrm{d} y\right| \lesssim 2^{k(n+|\gamma|)} G_{N} f(x), \\
x \in B_{k+2} .
\end{array}
$$

Since

$$
\left|\frac{\psi_{\gamma}^{k}(x) \chi_{\widetilde{A}_{k, \varepsilon}}(x)}{\left|\widetilde{A}_{k, \varepsilon}\right|}-\frac{\psi_{\gamma}^{k+1}(x) \chi_{\widetilde{A}_{k+1, \varepsilon}}(x)}{\left|\widetilde{A}_{k+1, \varepsilon}\right|}\right| \lesssim 2^{-k(n+|\gamma|)} \sum_{l=k-1}^{k+1} \chi_{l}(x),
$$

it follows that

$$
\left\|h_{k, \gamma}^{(j)}\right\|_{L^{p(\cdot)}} \leqslant C \sum_{l=k-1}^{k+1}\left\|\left(G_{N} f\right) \chi_{l}\right\|_{L^{p(\cdot)}} .
$$

Take $\widetilde{b}=C$. It is easy to show that each $a_{k, \gamma}^{(j)}$ is a central $(\alpha(\cdot)$, $p(\cdot))$-atom with support contained in $\widetilde{A}_{k, \varepsilon} \cup \widetilde{A}_{k+1, \varepsilon} \subset B_{k+2}$, and

$$
\alpha_{k, \gamma}=C \sum_{l=k-1}^{k+1}\left\|\left(G_{N} f\right) \chi_{l}\right\|_{L^{p(\cdot)}}\left|B_{k+2}\right|^{\alpha_{k+2} / n}
$$

where $C$ is a constant independent of $j, f, k$, and $\gamma$. Moreover, we have

$$
\begin{aligned}
& \sup _{L \in \mathbb{Z}} 2^{-L \lambda q} \sum_{k=-\infty}^{L} \sum_{|\gamma| \leqslant m}\left|\alpha_{k, \gamma}\right|^{q} \\
& \quad \leq \sup _{L \in \mathbb{Z}} 2^{-L \lambda q} \sum_{k=-\infty}^{L}\left|B_{k+2}\right|^{q \alpha_{k+2} / n}\left(\sum_{l=k-1}^{k+1}\left\|\left(G_{N} f\right) \chi_{l}\right\|_{L^{p(\cdot)}}\right)^{q} \\
& \quad \leq \sup _{L \in \mathbb{Z}} 2^{-L \lambda q} \sum_{k=-\infty}^{L}\left|B_{k+2}\right|^{q \alpha_{k+2} / n}\left\|\left(G_{N} f\right) \chi_{k}\right\|_{L^{p(\cdot)}}^{q} \\
& :=B .
\end{aligned}
$$

Using the same argument as before for $A$, we obtain

$$
B \lesssim\left\|G_{N} f\right\|_{M \dot{K}_{p(\cdot), \lambda}^{\alpha(\cdot), q}}^{q}
$$

Therefore,

$$
\sup _{L \in \mathbb{Z}} 2^{-L \lambda q} \sum_{k=-\infty}^{L}\left|\sum_{|\gamma| \leqslant m} \alpha_{k, \gamma}\right|^{q} \lesssim\left\|G_{N} f\right\|_{M K_{p(\cdot), \lambda}^{\alpha(\cdot), q}}^{p} .
$$

Thus, we obtain that

$$
f^{(j)}(x)=\sum_{d=-\infty}^{\infty} \lambda_{d} a_{d}^{(j)}(x)
$$

where each $a_{d}^{(j)}$ is a central $(\alpha(\cdot), p(\cdot))$-atom with support contained in $\widetilde{A}_{d, \varepsilon} \cup \widetilde{A}_{d+1, \varepsilon} \subset B_{d+2}, \lambda_{d}$ is independent of $j$ and

$$
\sup _{L \in \mathbb{Z}} 2^{-L \lambda}\left(\sum_{d=-\infty}^{L}\left|\lambda_{d}\right|^{q}\right)^{1 / q} \leqslant C\left\|G_{N} f\right\|_{M \dot{K}_{p(\cdot), \lambda}^{\alpha(\cdot), q}}<\infty
$$

where $C$ is independent of $j$ and $f$.

Since

$$
\sup _{j \in \mathbb{N}_{0}}\left\|a_{0}^{(j)}\right\|_{L^{p(\cdot)}} \leqslant\left|B_{2}\right|^{-\alpha_{2} / n}
$$

by the Banach-Alaoglu theorem we obtain a subsequence $\left\{a_{0}^{\left(j_{n_{0}}\right)}\right\}$ of $\left\{a_{0}^{(j)}\right\}$ converging in the weak $*$ topology of $L^{p(\cdot)}$ to some $a_{0} \in L^{p(\cdot)}$. It is easy to verify that $a_{0}$ is a central $(\alpha(\cdot)$, $p(\cdot))$-atom supported on $B_{2}$. Next, since

$$
\sup _{j_{n_{0}} \in \mathbb{N}_{0}}\left\|a_{1}^{\left(j_{n_{0}}\right)}\right\|_{L^{p(\cdot)}} \leqslant\left|B_{3}\right|^{-\alpha_{3} / n}
$$

another application of the Banach-Alaoglu theorem yields a subsequence $\left\{a_{1}^{\left(j_{n_{1}}\right)}\right\}$ of $\left\{a_{1}^{\left(j_{n_{0}}\right)}\right\}$ which converges weak $*$ in $L^{p(\cdot)}$ to a central $(\alpha(\cdot), p(\cdot))$-atom $a_{1}$ with support in $B_{3}$. Furthermore,

$$
\sup _{j_{n_{1}} \in \mathbb{N}_{0}}\left\|a_{-1}^{\left(j_{n_{1}}\right)}\right\|_{L^{p(\cdot)}} \leqslant\left|B_{1}\right|^{-\alpha_{1} / n} .
$$

Similarly, there exists a subsequence $\left\{a_{-1}^{\left(j_{n_{-1}}\right)}\right\}$ of $\left\{a_{-1}^{\left(j_{n_{1}}\right)}\right\}$ which converges weak $*$ in $L^{p(\cdot)}$ to some $a_{-1} \in L^{p(\cdot)}$, and $a_{-1}$ is a central $(\alpha(\cdot), p(\cdot))$-atom supported on $B_{1}$. Repeating the above procedure for each $d \in \mathbb{Z}$, we can find a subsequence $\left\{a_{d}^{\left(j_{n_{d}}\right)}\right\}$ of $\left\{a_{d}^{(j)}\right\}$ converging weak $*$ in $L^{p(\cdot)}$ to some $a_{d} \in L^{p(\cdot)}$ which is a central $(\alpha(\cdot), p(\cdot))$-atom supported on $B_{d+2}$. By using the diagonal method we obtain a subsequence $\left\{j_{\nu}\right\}$ of $\mathbb{N}_{0}$ such that, for each $d \in \mathbb{Z}, \lim _{v \rightarrow \infty} a_{d}^{\left(j_{v}\right)}=a_{d}$ in the weak $*$ topology of $L^{p(\cdot)}$ and therefore in $\mathcal{S}^{\prime}\left(\mathbb{R}^{n}\right)$.

Now we only need to prove that $f=\sum_{d=-\infty}^{\infty} \lambda_{d} a_{d}$ in the sense of $\mathcal{S}^{\prime}\left(\mathbb{R}^{n}\right)$. For each $\varphi \in \mathcal{S}\left(\mathbb{R}^{n}\right)$, note that supp $a_{d}^{\left(j_{\nu}\right)} \subset$ $\left(\widetilde{A}_{d, \varepsilon} \cup \widetilde{A}_{d+1, \varepsilon}\right) \subset\left(A_{d-1} \cup A_{d} \cup A_{d+1} \cup A_{d+2}\right)$. Using the same argument in [48], we have

$$
\langle f, \varphi\rangle=\lim _{\nu \rightarrow \infty} \sum_{d=-\infty}^{\infty} \lambda_{d} \int_{\mathbb{R}^{n}} a_{d}^{\left(j_{v}\right)}(x) \varphi(x) \mathrm{d} x .
$$


Recall that $m=\max \left\{\left[\alpha(0)-n \delta_{2}\right],\left[\alpha_{\infty}-n \delta_{2}\right]\right\}$. If $d \leqslant 0$, then by Lemmas 3 and 4 we have

$$
\begin{aligned}
& \left|\int_{\mathbb{R}^{n}} a_{d}^{\left(j_{\nu}\right)}(x) \varphi(x) \mathrm{d} x\right| \\
& \quad=\left|\int_{\mathbb{R}^{n}} a_{d}^{\left(j_{\nu}\right)}(x)\left(\varphi(x)-\sum_{|\beta| \leqslant m} \frac{D^{\beta} \varphi(0)}{\beta !} x^{\beta}\right) \mathrm{d} x\right| \\
& \quad \lesssim \int_{\mathbb{R}^{n}}\left|a_{d}^{\left(j_{\nu}\right)}(x)\right| \cdot|x|^{m+1} \mathrm{~d} x \\
& \quad \leqslant 2^{d(m+1)} \int_{\mathbb{R}^{n}}\left|a_{d}^{\left(j_{\nu}\right)}(x)\right| \mathrm{d} x \\
& \quad \leqslant 2^{d\left(m+1-\alpha_{d+2}\right)} \| \chi_{B_{d+2}}||_{L^{p^{\prime}(\cdot)}} \\
& \quad \leqslant 2^{d\left(m+1-\alpha_{d+2}\right)}\left(\frac{\left|B_{d+2}\right|}{\left|B_{2}\right|}\right)^{\delta_{2}}\left\|\chi_{B_{2}}\right\|_{L^{p^{\prime}(\cdot)}} \\
& \quad \leqslant 2^{d\left(m+1-\alpha_{d+2}+n \delta_{2}\right)} \frac{\left|B_{2}\right|}{\left|B_{0}\right|}\left\|\chi_{B_{0}}\right\|_{L^{p^{\prime}(\cdot)}} \\
& \quad \leqslant 2^{d\left(m+1-\alpha_{d+2}+n \delta_{2}\right)} .
\end{aligned}
$$

If $d>0$, let $k_{0} \in \mathbb{N}_{0}$ such that $\min \left\{k_{0}+\alpha(0)-n, k_{0}+\alpha_{\infty}-\right.$ $n\}>0$; then by Lemmas 4 and 3 again we have

$$
\begin{aligned}
\left|\int_{\mathbb{R}^{n}} a_{d}^{\left(j_{\nu}\right)}(x) \varphi(x) \mathrm{d} x\right| & \lesssim \int_{\mathbb{R}^{n}}\left|a_{d}^{\left(j_{\nu}\right)}(x)\right||x|^{-k_{0}} \mathrm{~d} x \\
& \lesssim 2^{-d\left(k_{0}+\alpha_{d+2}\right)}\left\|\chi_{B_{d+2}}\right\|_{L^{p^{\prime}(\cdot)}} \\
& \lesssim 2^{-d\left(k_{0}+\alpha_{d+2}\right)} \frac{\left|B_{d+2}\right|}{\left|B_{0}\right|}\left\|\chi_{B_{0}}\right\|_{L^{p^{(}(\cdot)}} \\
& \lesssim 2^{-d\left(k_{0}+\alpha_{d+2}-n\right)} .
\end{aligned}
$$

Let

$$
\mu_{d}= \begin{cases}\left|\lambda_{d}\right| 2^{d\left(m+1-\alpha_{d+2}+n \delta_{2}\right)}, & d \leqslant 0 \\ \left|\lambda_{d}\right| 2^{-d\left(k_{0}+\alpha_{d+2}-n\right)}, & d>0 .\end{cases}
$$

Then

$$
\begin{aligned}
& \sup _{L \in \mathbb{Z}} 2^{-L \lambda} \sum_{d=-\infty}^{L}\left|\mu_{d}\right| \lesssim\left(\sup _{L \in \mathbb{Z}} 2^{-L \lambda q} \sum_{d=-\infty}^{L}\left|\lambda_{d}\right|^{q}\right)^{1 / q} \\
& \leqslant\left\|G_{N} f\right\|_{M \dot{K}_{p(\cdot), \lambda}^{\alpha(\cdot), q}}<\infty \text {, } \\
& \left|\lambda_{d}\right|\left|\int_{\mathbb{R}^{n}} a_{d}^{\left(j_{y}\right)}(x) \varphi(x) \mathrm{d} x\right| \lesssim\left|\mu_{d}\right|,
\end{aligned}
$$

which implies that

$$
\begin{aligned}
\langle f, \varphi\rangle & =\sum_{d=-\infty}^{\infty} \lim _{\nu \rightarrow \infty} \lambda_{d} \int_{\mathbb{R}^{n}} a_{d}^{\left(j_{v}\right)}(x) \varphi(x) \mathrm{d} x \\
& =\sum_{d=-\infty}^{\infty} \lambda_{d} \int_{\mathbb{R}^{n}} a_{d}(x) \varphi(x) \mathrm{d} x .
\end{aligned}
$$

This establishes the identity we wanted.
To prove the sufficiency, for convenience, we denote $\sup _{L \in \mathbb{Z}} 2^{-L \lambda} \sum_{k=-\infty}^{L}\left|\lambda_{k}\right|^{q}=\Lambda$. Firstly we have

$$
\begin{aligned}
& \left\|G_{N} f\right\|_{M \dot{K}_{p(\cdot), \lambda}^{\alpha(\cdot), q}}^{q} \\
& \approx \max \left\{\sup _{L \leqslant 0, L \in \mathbb{Z}} 2^{-L \lambda q} \sum_{k=-\infty}^{L} 2^{k q \alpha(0)}\left\|\left(G_{N} f\right) \chi_{k}\right\|_{L^{p(\cdot)}}^{q},\right. \\
& \sup _{L>0, L \in \mathbb{Z}} 2^{-L \lambda q}\left(\sum_{k=-\infty}^{-1} 2^{k q \alpha(0)}\left\|\left(G_{N} f\right) \chi_{k}\right\|_{L^{p(\cdot)}}^{q}\right. \\
& \left.\left.+\sum_{k=0}^{L} 2^{k q \alpha(\infty)}\left\|\left(G_{N} f\right) \chi_{k}\right\|_{L^{p(\cdot)}}^{q}\right)\right\}
\end{aligned}
$$

$\lesssim \max \{I, I I+I I I\}$,

where

$$
\begin{gathered}
I:=\sup _{L \leqslant 0, L \in \mathbb{Z}} 2^{-L \lambda q} \sum_{k=-\infty}^{L} 2^{k q \alpha(0)}\left\|\left(G_{N} f\right) \chi_{k}\right\|_{L^{p \cdot()}}^{q} \\
I I:=\sum_{k=-\infty}^{-1} 2^{k q \alpha(0)}\left\|\left(G_{N} f\right) \chi_{k}\right\|_{L^{p(\cdot)}}^{q} \\
I I I:=\sup _{L>0, L \in \mathbb{Z}} 2^{-L \lambda q} \sum_{k=0}^{L} 2^{k q \alpha(\infty)}\left\|\left(G_{N} f\right) \chi_{k}\right\|_{L^{p(\cdot)}}^{q} .
\end{gathered}
$$

Now we have

$$
\begin{aligned}
I= & \sup _{L \leqslant 0, L \in \mathbb{Z}} 2^{-L \lambda q} \sum_{k=-\infty}^{L} 2^{k q \alpha(0)}\left\|\left(G_{N} f\right) \chi_{k}\right\|_{L^{p(\cdot)}}^{q} \\
\leq & \sup _{L \leqslant 0, L \in \mathbb{Z}} 2^{-L \lambda q} \sum_{k=-\infty}^{L} 2^{k q \alpha(0)}\left(\sum_{l=k}^{\infty}\left|\lambda_{l}\right|\left\|a_{l}\right\|_{L^{p(\cdot)}}\right)^{q} \\
& +\sup _{L \leqslant 0, L \in \mathbb{Z}} 2^{-L \lambda q} \sum_{k=-\infty}^{L} 2^{k q \alpha(0)}\left(\sum_{l=-\infty}^{k-1}\left|\lambda_{l}\right|\left\|\left(G_{N} a_{l}\right) \chi_{k}\right\|_{L^{p(\cdot)}}\right)^{q} \\
:= & I_{1}+I_{2} . \\
I I= & \sum_{k=-\infty}^{-1} 2^{k q \alpha(0)}\left\|\left(G_{N} f\right) \chi_{k}\right\|_{L^{p(\cdot)}}^{q} \\
\leq & \sum_{k=-\infty}^{-1} 2^{k q \alpha(0)}\left(\sum_{l=k}^{\infty}\left|\lambda_{l}\right|\left\|a_{l}\right\|_{L^{p(\cdot)}}\right)^{q} \\
& +\sum_{k=-\infty}^{-1} 2^{k q \alpha(0)}\left(\sum_{l=-\infty}^{k-1}\left|\lambda_{l}\right|\left\|\left(G_{N} a_{l}\right) \chi_{k}\right\|_{L^{p(\cdot)}}\right)^{q} \\
:= & I I_{1}+I I_{2} \\
I I I= & \sup _{L>0, L \in \mathbb{Z}} 2^{-L \lambda q} \sum_{k=0}^{L} 2^{k q \alpha_{\infty}}\left\|\left(G_{N} f\right) \chi_{k}\right\|_{L^{p(\cdot)}}^{q} \\
\leq & \sup _{L>0, L \in \mathbb{Z}} 2^{-L \lambda q} \sum_{k=0}^{L} 2^{k q \alpha_{\infty}}\left(\sum_{l=k}^{L}\left|\lambda_{l}\right|\left\|a_{l}\right\|_{L^{p(\cdot)}}\right)^{q}
\end{aligned}
$$




$$
\begin{aligned}
& \quad+\sup _{L>0, L \in \mathbb{Z}} 2^{-L \lambda q} \sum_{k=0}^{L} 2^{k q \alpha_{\infty}}\left(\sum_{l=-\infty}^{k-1}\left|\lambda_{l}\right|\left\|\left(G_{N} a_{l}\right) \chi_{k}\right\|_{L^{p(\cdot)}}\right)^{q} \\
& :=I I I_{1}+I I I_{2} .
\end{aligned}
$$

To estimate $I, I I$, and $I I I$ we need a pointwise estimate for $G_{N} a_{l}(x)$ on $D_{k}$, where $k \geqslant l+2$. Let $\phi \in \mathscr{A}_{N}, m \in \mathbb{N}$ such that $\alpha_{k}-n \delta_{2}<m+1$. Denote by $P_{m}$ the $m$ th order Taylor series expansion of $\phi$ at $y / t$. If $|x-y|<t$, then from the vanishing moment condition of $a_{l}$ we have

$$
\begin{aligned}
& \left|a_{l} * \phi_{t}(y)\right| \\
& \quad=t^{-n}\left|\int_{\mathbb{R}^{n}} a_{l}(z)\left(\phi\left(\frac{y-z}{t}\right)-P_{m}\left(-\frac{z}{t}\right)\right) \mathrm{d} z\right| \\
& \quad \leq t^{-n} \int_{\mathbb{R}^{n}}\left|a_{l}(z)\right|\left|\frac{z}{t}\right|^{m+1}\left(1+\left|\frac{(y-\theta z)}{t}\right|\right)^{-(n+m+1)} \mathrm{d} z \\
& \quad \leq \int_{\mathbb{R}^{n}}\left|a_{l}(z)\right||z|^{m+1}(t+|y-\theta z|)^{-(n+m+1)} \mathrm{d} z,
\end{aligned}
$$

where $0<\theta<1$. Since $x \in D_{k}$ for $k \in \mathbb{Z}$, we have $|x| \geqslant 2^{k-1}$. From $|x-y|<t$ and $|z|<2^{l}$, we have

$$
t+|y-\theta z| \geqslant|x-y|+|y-\theta z| \geqslant|x|-|z| \geqslant \frac{|x|}{2} .
$$

Thus,

$$
\begin{aligned}
& \left|a_{l} * \phi_{t}(y)\right| \\
& \quad \lesssim \int_{\mathbb{R}^{n}}\left|a_{l}(z)\right||z|^{m+1}(|x-y|+|y-\theta z|)^{-(n+m+1)} \mathrm{d} z \\
& \quad \lesssim 2^{l(m+1)}|x|^{-(n+m+1)} \int_{\mathbb{R}^{n}}|a(z)| \mathrm{d} z \\
& \quad \lesssim 2^{l(m+1)} 2^{-k(n+m+1)}\left|B_{l}\right|^{-\alpha_{l} / n}\left\|\chi_{B_{l}}\right\|_{L^{p^{\prime}(\cdot)}} .
\end{aligned}
$$

Therefore, we have

$$
\begin{array}{r}
G_{N} a_{l}(x) \lesssim 2^{l(m+1)} 2^{-k(n+m+1)}\left|B_{l}\right|^{-\alpha_{l} / n}\left\|\chi_{B_{l}}\right\|_{L^{p^{\prime}(\cdot)}}, \\
x \in D_{k}, \quad k \geqslant l+2 .
\end{array}
$$

To proceed, we consider them into two cases $0<q \leqslant 1$ and $1<q<\infty$.

If $0<q \leqslant 1$,

$$
\begin{aligned}
& I_{1}=\sup _{L \leqslant 0, L \in \mathbb{Z}} 2^{-L \lambda q} \sum_{k=-\infty}^{L} 2^{\alpha(0) k q}\left(\sum_{l=k}^{\infty}\left|\lambda_{l}\right|\left\|\left(T b_{l}\right) \chi_{k}\right\|_{L^{p(\cdot)}}\right)^{q} \\
& \vdots \sup _{L \leqslant 0, L \in \mathbb{Z}} 2^{-L \lambda q} \sum_{k=-\infty}^{L} 2^{\alpha(0) k q}\left(\sum_{l=k}^{\infty}\left|\lambda_{l}\right| 2^{-\alpha_{l} l}\right)^{q} \\
& \leqslant \sup _{L \leqslant 0, L \in \mathbb{Z}} 2^{-L \lambda q} \sum_{k=-\infty}^{L} 2^{\alpha(0) k q} \\
& \quad \times\left(\sum_{l=k}^{-1}\left|\lambda_{l}\right|^{q} 2^{-\alpha(0) l q}+\sum_{l=0}^{\infty}\left|\lambda_{l}\right|^{q} 2^{-\alpha_{\infty} l q}\right)
\end{aligned}
$$

$$
\begin{aligned}
& \lesssim \sup _{L \leqslant 0, L \in \mathbb{Z}} 2^{-L \lambda q} \sum_{k=-\infty}^{L} \sum_{l=k}^{-1}\left|\lambda_{l}\right|^{q} 2^{\alpha(0)(k-l) q} \\
& +\sup _{L \leqslant 0, L \in \mathbb{Z}} 2^{-L \lambda q} \sum_{k=-\infty}^{L} 2^{\alpha(0) k q} \sum_{l=0}^{\infty}\left|\lambda_{l}\right|^{q} 2^{-\alpha_{\infty} l q} \\
& \lesssim \sup _{L \leqslant 0, L \in \mathbb{Z}} 2^{-L \lambda q} \sum_{l=-\infty}^{-1}\left|\lambda_{l}\right|^{q} \sum_{k=-\infty}^{l} 2^{\alpha(0)(k-l) q} \\
& +\sup _{L \leqslant 0, L \in \mathbb{Z}} \sum_{l=0}^{\infty} 2^{-l \lambda q}\left|\lambda_{l}\right|^{q} 2^{\left(\lambda-\alpha_{\infty}\right) l q} 2^{-L \lambda q} \sum_{k=-\infty}^{L} 2^{\alpha(0) k q} \\
& \lesssim \Lambda+\sup _{L \leqslant 0, L \in \mathbb{Z}} 2^{-L \lambda q} \sum_{l=L}^{-1}\left|\lambda_{l}\right|^{q} \sum_{k=-\infty}^{l} 2^{\alpha(0)(k-l) q} \\
& +\Lambda \sup _{L \leqslant 0, L \in \mathbb{Z}} \sum_{l=0}^{\infty} 2^{\left(\lambda-\alpha_{\infty}\right) l q} \sum_{k=-\infty}^{L} 2^{\alpha(0) k q-L \lambda q} \\
& \lesssim \Lambda+\sup _{L \leqslant 0, L \in \mathbb{Z}} \sum_{l=L}^{-1} 2^{-l \lambda q}\left|\lambda_{l}\right|^{q} 2^{(l-L) \lambda q} \sum_{k=-\infty}^{l} 2^{\alpha(0)(k-l) q}+\Lambda \\
& \lesssim \Lambda+\Lambda \sup _{L \leqslant 0, L \in \mathbb{Z} l=L} \sum_{k=-\infty}^{-1} 2^{(l-L) \lambda q} \sum_{k}^{l} 2^{\alpha(0)(k-l) q}
\end{aligned}
$$$$
\lesssim \Lambda \text {. }
$$$$
\begin{aligned}
& I_{2}= \sup _{L \leqslant 0, L \in \mathbb{Z}} 2^{-L \lambda q} \sum_{k=-\infty}^{L} 2^{k q \alpha(0)}\left(\sum_{l=-\infty}^{k-1}\left|\lambda_{l}\right|\left\|\left(G_{N} a_{l}\right) \chi_{k}\right\|_{L^{p(\cdot)}}\right)^{q} \\
& \leqslant \sup _{L \leqslant 0, L \in \mathbb{Z}} 2^{-L \lambda q} \\
& \quad \times \sum_{k=-\infty}^{L} 2^{k q \alpha(0)}\left(\sum_{l=-\infty}^{k-1}\left|\lambda_{l}\right| 2^{l(m+1)-k(n+m+1)}\right. \\
&\left.\quad \times\left|B_{l}\right|^{-\alpha_{l} / n}\left\|\chi_{B_{l}}\right\|_{L^{p^{\prime}(\cdot)}}\left\|\chi_{B_{k}}\right\|_{L^{p(\cdot)}}\right)^{q}
\end{aligned}
$$$$
\lesssim \sup _{L \leqslant 0, L \in \mathbb{Z}} 2^{-L \lambda q} \sum_{k=-\infty}^{L}\left(\sum_{l=-\infty}^{k-1}\left|\lambda_{l}\right| 2^{(l-k)\left(m+1+n \delta_{2}\right)-(l-k) \alpha(0)}\right)^{q}
$$$$
\lesssim \sup _{L \leqslant 0, L \in \mathbb{Z}} 2^{-L \lambda q} \sum_{k=-\infty}^{L}\left(\sum_{l=-\infty}^{k-1}\left|\lambda_{l}\right| 2^{(l-k)\left(m+1+n \delta_{2}-\alpha(0)\right)}\right)^{q}
$$$$
=\sup _{L \leqslant 0, L \in \mathbb{Z}} 2^{-L \lambda q} \sum_{l=-\infty}^{L-1} \sum_{k=l+1}^{L}\left|\lambda_{l}\right|^{q} 2^{(l-k)\left(m+1+n \delta_{2}-\alpha(0)\right) q}
$$$$
\lesssim \sup _{L \leqslant 0, L \in \mathbb{Z}} 2^{-L \lambda q} \sum_{l=-\infty}^{L}\left|\lambda_{l}\right|^{q}
$$

$\lesssim \Lambda$. 
Then we turn to estimate $I I$ :

$$
\begin{aligned}
I_{1} & =\sum_{k=-\infty}^{-1} 2^{k q \alpha(0)}\left(\sum_{l=k}^{\infty}\left|\lambda_{l}\right|\left\|a_{l}\right\|_{L^{p(\cdot)}}\right)^{q} \\
& \leqslant \sum_{k=-\infty}^{-1} 2^{k q \alpha(0)}\left(\sum_{l=k}^{\infty}\left|\lambda_{l}\right|\left|B_{l}\right|^{-\alpha_{l} / n}\right)^{q} \\
& \leqslant \sum_{k=-\infty}^{-1} 2^{\alpha(0) k q}\left(\sum_{l=k}^{-1}\left|\lambda_{l}\right|^{q} 2^{-\alpha(0) l q}+\sum_{l=0}^{\infty}\left|\lambda_{l}\right|^{q} 2^{-\alpha_{\infty} l q}\right) \\
& \vdots \sum_{k=-\infty}^{-1} \sum_{l=k}^{-1}\left|\lambda_{l}\right|^{q} 2^{\alpha(0)(k-l) q} \\
& +\sum_{k=-\infty}^{-1} 2^{\alpha(0) k q} \sum_{l=0}^{\infty}\left|\lambda_{l}\right|^{q} 2^{-\alpha_{\infty} l q} \\
& \lesssim \sum_{l=-\infty}^{-1}\left|\lambda_{l}\right|^{q} \sum_{k=-\infty}^{l} 2^{\alpha(0)(k-l) q} \\
& +\sum_{l=0}^{\infty}\left|\lambda_{l}\right|^{q} 2^{-\alpha_{\infty} l q} \sum_{k=-\infty}^{-1} 2^{\alpha(0) k q} \\
& \sum_{l=-\infty}^{-1}\left|\lambda_{l}\right|^{q}+\sum_{l=0}^{\infty} 2^{-l \lambda q}\left|\lambda_{l}\right|^{q} 2^{-\alpha_{\infty} l q} \sum_{k=-\infty}^{-1} 2^{\alpha(0) k q} \\
& \left.\vdots \lambda_{i}\right|^{q} \sum_{l=0}^{\infty} 2^{\left(\lambda-\alpha_{\infty}\right) l q} \sum_{k=-\infty}^{l} 2^{\alpha(0) k q}
\end{aligned}
$$$$
I I_{2}=\sum_{k=-\infty}^{-1} 2^{k q \alpha(0)}\left(\sum_{l=-\infty}^{k-1}\left|\lambda_{l}\right|\left\|\left(G_{N} a_{l}\right) \chi_{k}\right\|_{L^{p(\cdot)}}\right)^{q}
$$$$
\lesssim \sum_{k=-\infty}^{-1} 2^{k q \alpha(0)}\left(\sum_{l=-\infty}^{k-1}\left|\lambda_{l}\right| 2^{l(m+1)-k(n+m+1)}\right.
$$$$
\left.\times\left|B_{l}\right|^{-\alpha_{l} / n}\left\|\chi_{B_{l}}\right\|_{L^{p^{\prime}(\cdot)}}\left\|\chi_{B_{k}}\right\|_{L^{p(\cdot)}}\right)^{q}
$$$$
\lesssim \sum_{k=-\infty}^{-1}\left(\sum_{l=-\infty}^{k-1}\left|\lambda_{l}\right| 2^{(l-k)\left(m+1+n \delta_{2}\right)-(l-k) \alpha(0)}\right)^{q}
$$$$
\lesssim \sum_{k=-\infty}^{-1}\left(\sum_{l=-\infty}^{k-1}\left|\lambda_{l}\right| 2^{(l-k)\left(m+1+n \delta_{2}-\alpha(0)\right)}\right)^{q}
$$$$
\lesssim \sum_{k=-\infty}^{-1}\left(\sum_{l=-\infty}^{k-1}\left|\lambda_{l}\right|^{q} 2^{(l-k)\left(m+1+n \delta_{2}-\alpha(0)\right) q}\right)
$$$$
=\sum_{l=-\infty}^{-2} \sum_{k=l+1}^{-1}\left|\lambda_{l}\right|^{q} 2^{(l-k)\left(m+1+n \delta_{2}-\alpha(0)\right) q}
$$$$
\lesssim \sum_{l=-\infty}^{-1}\left|\lambda_{l}\right|^{q} \leqslant \Lambda
$$

Third, we estimate $I I I$ :

$$
\begin{aligned}
& I I I_{1}=\sup _{L>0, L \in \mathbb{Z}} 2^{-L \lambda q} \sum_{k=0}^{L} 2^{k q \alpha_{\infty}}\left|B_{k}\right|^{\alpha_{\infty} q / n}\left(\sum_{l=k}^{\infty}\left|\lambda_{l}\right|\left\|a_{l}\right\|_{L^{p(\cdot)}}\right)^{q} \\
& \lesssim \sup _{L>0, L \in \mathbb{Z}} 2^{-L \lambda q} \sum_{k=0}^{L} 2^{k q \alpha_{\infty}}\left|B_{k}\right|^{\alpha_{\infty} q / n}\left(\sum_{l=k}^{\infty}\left|\lambda_{l}\right|\left|B_{l}\right|^{-\alpha_{l} / n}\right)^{q} \\
& \lesssim \sup _{L>0, L \in \mathbb{Z}} 2^{-L \lambda q} \sum_{k=0}^{L} \sum_{l=k}^{\infty}\left|B_{k}\right|^{\alpha_{\infty} q / n}\left|\lambda_{l}\right|^{q}\left|B_{l}\right|^{-\alpha_{\infty} q / n} \\
& \lesssim \sup _{L>0, L \in \mathbb{Z}} 2^{-L \lambda q} \sum_{k=0}^{L} \sum_{l=k}^{\infty}\left|\lambda_{l}\right|^{q} 2^{(k-l) \alpha_{\infty} q} \\
& =\sup _{L>0, L \in \mathbb{Z}} 2^{-L \lambda q} \\
& \times\left[\sum_{l=0}^{L}\left|\lambda_{l}\right|^{q} \sum_{k=0}^{l} 2^{(k-l) \alpha_{\infty} q}+\sum_{l=L}^{\infty}\left|\lambda_{l}\right|^{q} \sum_{k=0}^{L} 2^{(k-l) \alpha_{\infty} q}\right] \\
& \lesssim \sup _{L>0, L \in \mathbb{Z}} 2^{-L \lambda q} \sum_{l=0}^{L}\left|\lambda_{l}\right|^{q} \\
& +\sup _{L>0, L \in \mathbb{Z}} \sum_{l=L}^{\infty} 2^{(l \lambda q-L \lambda q)} 2^{-l \lambda q} \sum_{i=-\infty}^{l}\left|\lambda_{i}\right|^{q} \sum_{k=0}^{L} 2^{(k-l) \alpha_{\infty} q / 2} \\
& \lesssim \Lambda+\Lambda \sup _{L>0, L \in \mathbb{Z}} \sum_{l=L}^{\infty} 2^{(l-L) \lambda q} 2^{(L-l) \alpha_{\infty} q / 2} \\
& \lesssim \Lambda+\Lambda \sup _{L>0, L \in \mathbb{Z}} \sum_{l=L}^{\infty} 2^{(l-L) q\left(\lambda-\alpha_{\infty} / 2\right)} \\
& \lesssim \Lambda \text {. } \\
& I I I_{2}=\sup _{L>0, L \in \mathbb{Z}} 2^{-L \lambda q} \sum_{k=0}^{L} 2^{k q \alpha_{\infty}}\left(\sum_{l=-\infty}^{k-1}\left|\lambda_{l}\right|\left\|\left(G_{N} a_{l}\right) \chi_{k}\right\|_{L^{p(\cdot)}}\right)^{q} \\
& \lesssim \sup _{L>0, L \in \mathbb{Z}} 2^{-L \lambda q} \\
& \times \sum_{k=0}^{L} 2^{k q \alpha_{\infty}}\left(\sum_{l=-\infty}^{k-1}\left|\lambda_{l}\right| 2^{l(m+1)-k(n+m+1)}\right. \\
& \left.\times\left|B_{l}\right|^{-\alpha_{l} / n}\left\|\chi_{B_{l}}\right\|_{L^{p^{\prime}(\cdot)}}\left\|\chi_{B_{k}}\right\|_{L^{p(\cdot)}}\right)^{q} \\
& \lesssim \sup _{L>0, L \in \mathbb{Z}} 2^{-L \lambda q} \sum_{k=0}^{L}\left(\sum_{l=-\infty}^{k-1}\left|\lambda_{l}\right| 2^{(l-k)\left(m+1+n \delta_{2}\right)-l \alpha_{l}+k \alpha_{\infty}}\right)^{q} \\
& \lesssim \sup _{L>0, L \in \mathbb{Z}} 2^{-L \lambda q} \sum_{k=0}^{L}\left(\sum_{l=-\infty}^{-1}\left|\lambda_{l}\right| 2^{(l-k)\left(m+1+n \delta_{2}\right)-l \alpha(0)+k \alpha_{\infty}}\right)^{q} \\
& +\sup _{L>0, L \in \mathbb{Z}} 2^{-L \lambda q} \sum_{k=0}^{L}\left(\sum_{l=0}^{k-1}\left|\lambda_{l}\right| 2^{(l-k)\left(m+1+n \delta_{2}-\alpha_{\infty}\right)}\right)^{q}
\end{aligned}
$$




$$
\begin{aligned}
& \lesssim \sup _{L>0, L \in \mathbb{Z}} 2^{-L \lambda q} \sum_{k=0}^{L} 2^{q k\left[\alpha_{\infty}-\left(m+1+n \delta_{2}\right)\right]} \\
& \quad \times\left(\sum_{l=-\infty}^{-1}\left|\lambda_{l}\right| 2^{l\left(m+1+n \delta_{2}-\alpha(0)\right)}\right)^{q} \\
& +\sup _{L>0, L \in \mathbb{Z}} 2^{-L \lambda q} \sum_{k=0}^{L} \sum_{l=0}^{k-1}\left|\lambda_{l}\right|^{q} 2^{(l-k)\left(m+1+n \delta_{2}-\alpha_{\infty}\right) q} \\
& \lesssim \sup _{L>0, L \in \mathbb{Z}} 2^{-L \lambda q} \sum_{l=-\infty}^{-1}\left|\lambda_{l}\right|^{q} 2^{l\left(m+1+n \delta_{2}-\alpha(0)\right) q} \\
& +\sup _{L>0, L \in \mathbb{Z}} 2^{-L \lambda q} \sum_{l=0}^{L-1} \sum_{k=l+1}^{L}\left|\lambda_{l}\right|^{q} 2^{(l-k)\left(m+1+n \delta_{2}-\alpha_{\infty}\right) q} \\
& \lesssim \sup _{L>0, L \in \mathbb{Z}} 2^{-L \lambda q} \sum_{l=-\infty}^{-1}\left|\lambda_{l}\right|^{q}+\sup _{L>0, L \in \mathbb{Z}} 2^{-L \lambda q} \sum_{l=0}^{L}\left|\lambda_{l}\right|^{q} \\
& =\sup _{L>0, L \in \mathbb{Z}} 2^{-L \lambda q} \sum_{l=-\infty}^{L}\left|\lambda_{l}\right|^{q} .
\end{aligned}
$$

$$
\begin{aligned}
& \lesssim \sup _{L \leqslant 0, L \in \mathbb{Z}} 2^{-L \lambda q} \sum_{l=-\infty}^{-1}\left|\lambda_{l}\right|^{q} \sum_{k=-\infty}^{l} 2^{\alpha(0)(k-l) q / 2} \\
& +\sup _{L \leqslant 0, L \in \mathbb{Z}} \sum_{l=0}^{\infty} 2^{-l \lambda q}\left|\lambda_{l}\right|^{q} 2^{\left(\lambda-\alpha_{\infty} / 2\right) l q} 2^{-L \lambda q} \sum_{k=-\infty}^{L} 2^{\alpha(0) k q} \\
& \leqslant \sup _{L \leqslant 0, L \in \mathbb{Z}} 2^{-L \lambda q} \sum_{l=-\infty}^{L}\left|\lambda_{l}\right|^{q} \\
& +\sup _{L \leqslant 0, L \in \mathbb{Z}} 2^{-L \lambda q} \sum_{l=L}^{-1}\left|\lambda_{l}\right|^{q} \sum_{k=-\infty}^{l} 2^{\alpha(0)(k-l) q / 2} \\
& +\Lambda \sup _{L \leqslant 0, L \in \mathbb{Z}} \sum_{l=0}^{\infty} 2^{\left(\lambda-\alpha_{\infty} / 2\right) l q} \sum_{k=-\infty}^{L} 2^{\alpha(0) k q-L \lambda q} \\
& \leqslant \Lambda+\sup _{L \leqslant 0, L \in \mathbb{Z}_{l=L}} \sum^{-1} 2^{-l \lambda q}\left|\lambda_{l}\right|^{q} 2^{(l-L) \lambda q} \\
& \quad \times \sum_{k=-\infty}^{l} 2^{\alpha(0)(k-l) q / 2}+\Lambda \\
& \leqslant \Lambda+\Lambda \sup _{L \leqslant 0, L \in \mathbb{Z} l=L} \sum_{l=-\infty} 2^{(l-L) \lambda q} \sum_{k=-\infty}^{j} 2^{\alpha(0)(k-l) q / 2} \\
& \leqslant \Lambda .
\end{aligned}
$$

If $1<q<\infty$, we have

$$
\begin{aligned}
& I_{1}=\sup _{L \leqslant 0, L \in \mathbb{Z}} 2^{-L \lambda q} \sum_{k=-\infty}^{L} 2^{k q \alpha(0)}\left(\sum_{l=k}^{\infty}\left|\lambda_{l}\right|\left\|a_{l}\right\|_{L^{p(\cdot)}}\right)^{q} \\
& \lesssim \sup _{L \leqslant 0, L \in \mathbb{Z}} 2^{-L \lambda q} \sum_{k=-\infty}^{L} 2^{k q \alpha(0)}\left(\sum_{l=k}^{\infty}\left|\lambda_{l}\right|\left|B_{l}\right|^{-\alpha_{l} / n}\right)^{q} \\
& \lesssim \sup _{L \leqslant 0, L \in \mathbb{Z}} 2^{-L \lambda q} \sum_{k=-\infty}^{L}\left(\sum_{l=k}^{-1}\left|\lambda_{l}\right| 2^{\alpha(0)(k-l)}\right)^{q} \\
& +\sup _{L \leqslant 0, L \in \mathbb{Z}} 2^{-L \lambda q} \sum_{k=-\infty}^{L} 2^{\alpha(0) k q}\left(\sum_{l=0}^{\infty}\left|\lambda_{l}\right| 2^{-\alpha_{\infty} l}\right)^{q} \\
& \lesssim \sup _{L \leqslant 0, L \in \mathbb{Z}} 2^{-L \lambda q} \sum_{k=-\infty}^{L}\left(\sum_{l=k}^{-1}\left|\lambda_{l}\right|^{q} 2^{\alpha(0)(k-l) q / 2}\right) \\
& \times\left(\sum_{l=k}^{-1} 2^{\alpha(0)(k-l) q^{\prime} / 2}\right)^{q / q^{\prime}} \\
& +\sup _{L \leqslant 0, L \in \mathbb{Z}} 2^{-L \lambda q} \sum_{k=-\infty}^{L} 2^{\alpha(0) k q}\left(\sum_{l=0}^{\infty}\left|\lambda_{l}\right|^{q} 2^{-\alpha_{\infty} j q / 2}\right) \\
& \times\left(\sum_{l=0}^{\infty} 2^{-\alpha_{\infty} j q^{\prime} / 2}\right)^{q / q^{\prime}} \\
& \lesssim \sup _{L \leqslant 0, L \in \mathbb{Z}} 2^{-L \lambda q} \sum_{k=-\infty}^{L} \sum_{l=k}^{-1}\left|\lambda_{l}\right|^{q} 2^{\alpha(0)(k-l) q / 2} \\
& +\sup _{L \leqslant 0, L \in \mathbb{Z}} 2^{-L \lambda q} \sum_{k=-\infty}^{L} 2^{\alpha(0) k q} \sum_{l=0}^{\infty}\left|\lambda_{l}\right|^{q} 2^{-\alpha_{\infty} l q / 2}
\end{aligned}
$$$$
I_{2}=\sup _{L \leqslant 0, L \in \mathbb{Z}} 2^{-L \lambda q} \sum_{k=-\infty}^{L} 2^{k q \alpha(0)}\left(\sum_{l=-\infty}^{k-1}\left|\lambda_{l}\right|\left\|\left(G_{N} a_{l}\right) \chi_{k}\right\|_{L^{p(\cdot)}}\right)^{q}
$$$$
\lesssim \sup _{L \leqslant 0, L \in \mathbb{Z}} 2^{-L \lambda q}
$$$$
\times \sum_{k=-\infty}^{L} 2^{k q \alpha(0)}\left(\sum_{l=-\infty}^{k-1}\left|\lambda_{l}\right| 2^{l(m+1)-k(n+m+1)}\right.
$$$$
\left.\times\left|B_{l}\right|^{-\alpha_{l} / n}\left\|\chi_{B_{l}}\right\|_{L^{p^{(\cdot)}}}\left\|\chi_{B_{k}}\right\|_{L^{p(\cdot)}}\right)^{q}
$$$$
\lesssim \sup _{L \leqslant 0, L \in \mathbb{Z}} 2^{-L \lambda q} \sum_{k=-\infty}^{L}\left(\sum_{l=-\infty}^{k-1}\left|\lambda_{l}\right| 2^{(l-k)\left(m+1+n \delta_{2}\right)-(l-k) \alpha(0)}\right)^{q}
$$$$
\lesssim \sup _{L \leqslant 0, L \in \mathbb{Z}} 2^{-L \lambda q} \sum_{k=-\infty}^{L}\left(\sum_{l=-\infty}^{k-1}\left|\lambda_{l}\right| 2^{(l-k)\left(m+1+n \delta_{2}-\alpha(0)\right)}\right)^{q}
$$$$
\lesssim \sup _{L>0, L \in \mathbb{Z}} 2^{-L \lambda q} \sum_{k=-\infty}^{L}\left(\sum_{l=-\infty}^{k-1}\left|\lambda_{l}\right|^{q} 2^{(l-k)\left(m+1+n \delta_{2}-\alpha(0)\right) q / 2}\right)
$$$$
\times\left(\sum_{l=-\infty}^{k-1} 2^{(l-k)\left(m+1+n \delta_{2}-\alpha(0)\right) q^{\prime} / 2}\right)^{q / q^{\prime}}
$$$$
\lesssim \sup _{L \leqslant 0, L \in \mathbb{Z}} 2^{-L \lambda q} \sum_{k=-\infty}^{L}\left(\sum_{l=-\infty}^{k-1}\left|\lambda_{l}\right|^{q} 2^{(l-k)\left(m+1+n \delta_{2}-\alpha(0)\right) q / 2}\right)
$$$$
=\sup _{L \leqslant 0, L \in \mathbb{Z}} 2^{-L \lambda q} \sum_{l=-\infty}^{L-1} \sum_{k=l+1}^{L}\left|\lambda_{l}\right|^{q} 2^{(l-k)\left(m+1+n \delta_{2}-\alpha(0)\right) q / 2}
$$$$
\lesssim \sup _{L \leqslant 0, L \in \mathbb{Z}} 2^{-L \lambda q} \sum_{l=-\infty}^{L}\left|\lambda_{l}\right|^{q}
$$$$
\leqslant \Lambda \text {. }
$$ 
Second, we estimate II. As the same argument before, we obtain that

$$
\begin{aligned}
& I I_{1}=\sum_{k=-\infty}^{-1} 2^{k q \alpha(0)}\left(\sum_{l=k}^{\infty}\left|\lambda_{l}\right|\left\|a_{l}\right\|_{L^{p(\cdot)}}\right)^{q} \\
& \lesssim \sum_{k=-\infty}^{-1} 2^{k q \alpha(0)}\left(\sum_{l=k}^{\infty}\left|\lambda_{l}\right|\left|B_{l}\right|^{-\alpha_{l} / n}\right)^{q} \\
& \lesssim \sum_{k=-\infty}^{-1}\left(\sum_{j=k}^{-1}\left|\lambda_{j}\right| 2^{\alpha(0)(k-j)}\right)^{q} \\
& +\sum_{k=-\infty}^{-1} 2^{\alpha(0) k q}\left(\sum_{j=0}^{\infty}\left|\lambda_{j}\right| 2^{-\alpha_{\infty} j}\right)^{q} \\
& \lesssim \sum_{k=-\infty}^{-1}\left(\sum_{l=k}^{-1}\left|\lambda_{l}\right|^{q} 2^{\alpha(0)(k-l) q / 2}\right)\left(\sum_{l=k}^{-1} 2^{\alpha(0)(k-l) q^{\prime} / 2}\right)^{q / q^{\prime}} \\
& +\sum_{k=-\infty}^{-1} 2^{\alpha(0) k q}\left(\sum_{l=0}^{\infty}\left|\lambda_{j}\right|^{q} 2^{-\alpha_{\infty} l q / 2}\right)\left(\sum_{l=0}^{\infty} 2^{-\alpha_{\infty} l q^{\prime} / 2}\right)^{q / q^{\prime}} \\
& \lesssim \sum_{k=-\infty}^{-1}\left|\lambda_{l}\right|^{q} \sum_{k=-\infty}^{l} 2^{\alpha(0)(k-l) q / 2} \\
& +\sum_{l=0}^{\infty}\left|\lambda_{l}\right|^{q} 2^{-\alpha_{\infty} l q / 2} \sum_{k=-\infty}^{-1} 2^{\alpha(0) k q} \\
& \lesssim \sum_{l=-\infty}^{-1}\left|\lambda_{l}\right|^{q} \\
& +\sum_{l=0}^{\infty} 2^{\left(\lambda-\alpha_{\infty} / 2\right) l q} 2^{-l \lambda q} \sum_{i=-\infty}^{l}\left|\lambda_{i}\right|^{q} \sum_{k=-\infty}^{-1} 2^{\alpha(0) k q} \\
& \leqslant \Lambda+\Lambda \sum_{l=0}^{\infty} 2^{\left(\lambda-\alpha_{\infty} / 2\right) l q} \sum_{k=-\infty}^{-1} 2^{\alpha(0) k q}
\end{aligned}
$$$$
\lesssim \Lambda \text {. }
$$$$
\begin{aligned}
I I_{2} & =\sum_{k=-\infty}^{-1} 2^{k q \alpha(0)}\left(\sum_{l=-\infty}^{k-1}\left|\lambda_{l}\right|\left\|\left(G_{N} a_{l}\right) \chi_{k}\right\|_{L^{p(\cdot)}}\right)^{q} \\
& \lesssim \sum_{k=-\infty}^{-1} 2^{k q \alpha(0)}\left(\sum_{l=-\infty}^{k-1}\left|\lambda_{l}\right| 2^{l(m+1)-k(n+m+1)}\right.
\end{aligned}
$$$$
\left.\times\left|B_{l}\right|^{-\alpha_{l} / n}\left\|\chi_{B_{l}}\right\|_{L^{p^{\prime}(\cdot)}}\left\|\chi_{B_{k}}\right\|_{L^{p(\cdot)}}\right)^{q}
$$$$
\lesssim \sum_{k=-\infty}^{-1}\left(\sum_{l=-\infty}^{k-1}\left|\lambda_{l}\right| 2^{(l-k)\left(m+1+n \delta_{2}\right)-(l-k) \alpha(0)}\right)^{q}
$$$$
\lesssim \sum_{k=-\infty}^{-1}\left(\sum_{l=-\infty}^{k-1}\left|\lambda_{l}\right| 2^{(l-k)\left(m+1+n \delta_{2}-\alpha(0)\right)}\right)^{q}
$$

$$
\begin{aligned}
& \lesssim \sum_{k=-\infty}^{-1}\left(\sum_{l=-\infty}^{k-1}\left|\lambda_{l}\right|^{q} 2^{(l-k)\left(m+1+n \delta_{2}-\alpha(0)\right) q / 2}\right) \\
& \times\left(\sum_{l=-\infty}^{k-1} 2^{(l-k)\left(m+1+n \delta_{2}-\alpha(0)\right) q^{\prime} / 2}\right)^{q / q^{\prime}} \\
& \leqslant \sum_{k=-\infty}^{-1}\left(\sum_{l=-\infty}^{k-1}\left|\lambda_{l}\right|^{q} 2^{(l-k)\left(m+1+n \delta_{2}-\alpha(0)\right) q / 2}\right) \\
& =\sum_{l=-\infty}^{-2} \sum_{k=l+1}^{-1}\left|\lambda_{l}\right|^{q} 2^{(l-k)\left(m+1+n \delta_{2}-\alpha(0)\right) q / 2} \\
& \leqslant \sum_{l=-\infty}^{-1}\left|\lambda_{l}\right|^{q} \\
& \leqslant \Lambda .
\end{aligned}
$$

Third, we estimate III. We have

$$
\begin{aligned}
& I I I_{1}=\sup _{L>0, L \in \mathbb{Z}} 2^{-L \lambda q} \sum_{k=0}^{L} 2^{k q \alpha_{\infty}}\left|B_{k}\right|^{\alpha_{\infty} q / n}\left(\sum_{l=k}^{\infty}\left|\lambda_{l}\right|\left\|a_{l}\right\|_{L^{p(\cdot)}}\right)^{q} \\
& \lesssim \sup _{L>0, L \in \mathbb{Z}} 2^{-L \lambda q} \sum_{k=0}^{L} 2^{k q \alpha_{\infty}}\left|B_{k}\right|^{\alpha_{\infty} q / n}\left(\sum_{l=k}^{\infty}\left|\lambda_{l}\right|\left|B_{l}\right|^{-\alpha_{l} / n}\right)^{q} \\
& \lesssim \sup _{L>0, L \in \mathbb{Z}} 2^{-L \lambda q} \sum_{k=0}^{L} 2^{k q \alpha_{\infty}}\left|B_{k}\right|^{\alpha_{\infty} q / n}\left(\sum_{l=k}^{\infty}\left|\lambda_{l}\right|^{q}\left|B_{l}\right|^{-\alpha_{l} q /(2 n)}\right) \\
& \times\left(\sum_{l=k}^{\infty}\left|B_{l}\right|^{-\alpha_{l} q^{\prime} /(2 n)}\right)^{q / q^{\prime}} \\
& \lesssim \sup _{L>0, L \in \mathbb{Z}} 2^{-L \lambda q} \sum_{k=0}^{L} \sum_{l=k}^{\infty}\left|B_{k}\right|^{\alpha_{\infty} q /(2 n)}\left|\lambda_{l}\right|^{q}\left|B_{l}\right|^{-\alpha_{\infty} q /(2 n)} \\
& \lesssim \sup _{L>0, L \in \mathbb{Z}} 2^{-L \lambda q} \sum_{k=0}^{L} \sum_{l=k}^{\infty}\left|\lambda_{l}\right|^{q} 2^{(k-l) \alpha_{\infty} q / 2} \\
& =\sup _{L>0, L \in \mathbb{Z}} 2^{-L \lambda q}\left[\sum_{l=0}^{L}\left|\lambda_{l}\right|^{q} \sum_{k=0}^{l} 2^{(k-l) \alpha_{\infty} q / 2}\right. \\
& \left.+\sum_{l=L}^{\infty}\left|\lambda_{l}\right|^{q} \sum_{k=0}^{L} 2^{(k-l) \alpha_{\infty} q / 2}\right] \\
& \lesssim \sup _{L>0, L \in \mathbb{Z}} 2^{-L \lambda q} \sum_{l=0}^{L}\left|\lambda_{l}\right|^{q} \\
& +\sup _{L>0, L \in \mathbb{Z}} \sum_{l=L}^{\infty} 2^{(j \lambda q-L \lambda q)} 2^{-l \lambda q} \sum_{i=-\infty}^{l}\left|\lambda_{i}\right|^{q} \sum_{k=0}^{L} 2^{(k-l) \alpha_{\infty} q / 2} \\
& \lesssim \Lambda+\Lambda \sup _{L>0, L \in \mathbb{Z}} \sum_{l=L}^{\infty} 2^{(l-L) \lambda q} 2^{(L-l) \alpha_{\infty} q / 2} \\
& \leqslant \Lambda+\Lambda \sup _{L>0, L \in \mathbb{Z}} \sum_{l=L}^{\infty} 2^{(l-L) q\left(\lambda-\alpha_{\infty} / 2\right)} \\
& \lesssim \Lambda .
\end{aligned}
$$




$$
\begin{aligned}
& I I I_{2}=\sup _{L>0, L \in \mathbb{Z}} 2^{-L \lambda q} \sum_{k=0}^{L} 2^{k q \alpha_{\infty}}\left(\sum_{l=-\infty}^{k-1}\left|\lambda_{l}\right|\left\|\left(G_{N} a_{l}\right) \chi_{k}\right\|_{L^{p(\cdot)}}\right)^{q} \\
& \lesssim \sup _{L>0, L \in \mathbb{Z}} 2^{-L \lambda q} \\
& \times \sum_{k=0}^{L} 2^{k q \alpha_{\infty}}\left(\sum_{l=-\infty}^{k-1}\left|\lambda_{l}\right| 2^{l(m+1)-k(n+m+1)}\right. \\
& \left.\times\left|B_{l}\right|^{-\alpha_{l} / n}\left\|\chi_{B_{l}}\right\|_{L^{p^{\prime}(\cdot)}}\left\|\chi_{B_{k}}\right\|_{L^{p(\cdot)}}\right)^{q} \\
& \lesssim \sup _{L>0, L \in \mathbb{Z}} 2^{-L \lambda q} \sum_{k=0}^{L}\left(\sum_{l=-\infty}^{k-1}\left|\lambda_{l}\right| 2^{(l-k)\left(m+1+n \delta_{2}\right)-l \alpha_{l}+k \alpha_{\infty}}\right)^{q} \\
& \lesssim \sup _{L>0, L \in \mathbb{Z}} 2^{-L \lambda q} \sum_{k=0}^{L}\left(\sum_{l=-\infty}^{-1}\left|\lambda_{l}\right| 2^{(l-k)\left(m+1+n \delta_{2}\right)-l \alpha(0)+k \alpha_{\infty}}\right)^{q} \\
& +\sup _{L>0, L \in \mathbb{Z}} 2^{-L \lambda q} \sum_{k=0}^{L}\left(\sum_{l=0}^{k-1}\left|\lambda_{l}\right| 2^{(l-k)\left(m+1+n \delta_{2}-\alpha_{\infty}\right)}\right)^{q} \\
& \lesssim \sup _{L>0, L \in \mathbb{Z}} 2^{-L \lambda q} \sum_{k=0}^{L} 2^{q k\left[\alpha_{\infty}-\left(m+1+n \delta_{2}\right)\right]} \\
& \times\left(\sum_{l=-\infty}^{-1}\left|\lambda_{l}\right| 2^{l\left(m+1+n \delta_{2}-\alpha(0)\right)}\right)^{q} \\
& +\sup _{L>0, L \in \mathbb{Z}} 2^{-L \lambda q} \sum_{k=0}^{L}\left(\sum_{l=0}^{k-1}\left|\lambda_{l}\right|^{q} 2^{(l-k)\left(m+1+n \delta_{2}-\alpha_{\infty}\right) q / 2}\right) \\
& \times\left(\sum_{l=0}^{k-1} 2^{(l-k)\left(m+1+n \delta_{2}-\alpha_{\infty}\right) q^{\prime} / 2}\right)^{q / q^{\prime}} \\
& \lesssim \sup _{L>0, L \in \mathbb{Z}} 2^{-L \lambda q}\left(\sum_{l=-\infty}^{-1}\left|\lambda_{l}\right|^{q} 2^{l\left(m+1+n \delta_{2}-\alpha(0)\right) q / 2}\right) \\
& \times\left(\sum_{l=-\infty}^{-1} 2^{l\left(m+1+n \delta_{2}-\alpha(0)\right) q^{\prime} / 2}\right)^{q / q^{\prime}} \\
& +\sup _{L>0, L \in \mathbb{Z}} 2^{-L \lambda q} \sum_{k=0}^{L} \sum_{l=0}^{k-1}\left|\lambda_{l}\right|^{q} 2^{(l-k)\left(m+1+n \delta_{2}-\alpha_{\infty}\right) q / 2} \\
& \lesssim \sup _{L>0, L \in \mathbb{Z}} 2^{-L \lambda q} \sum_{l=-\infty}^{-1}\left|\lambda_{l}\right|^{q} 2^{l\left(m+1+n \delta_{2}-\alpha(0)\right) q / 2} \\
& +\sup _{L>0, L \in \mathbb{Z}} 2^{-L \lambda q} \sum_{l=0}^{L-1} \sum_{k=l+1}^{L}\left|\lambda_{l}\right|^{q} 2^{(l-k)\left(m+1+n \delta_{2}-\alpha_{\infty}\right) q / 2} \\
& \lesssim \sup _{L>0, L \in \mathbb{Z}} 2^{-L \lambda q} \sum_{l=-\infty}^{-1}\left|\lambda_{l}\right|^{q}+\sup _{L>0, L \in \mathbb{Z}} 2^{-L \lambda q} \sum_{l=0}^{L}\left|\lambda_{l}\right|^{q} \\
& =\sup _{L>0, L \in \mathbb{Z}} 2^{-L \lambda q} \sum_{l=-\infty}^{L}\left|\lambda_{l}\right|^{q} \\
& \leqslant \Lambda \text {. }
\end{aligned}
$$

\section{Applications}

As an application of the atomic decompositions, we will prove the following result.

Theorem 14. Let $0<q<\infty, 0 \leq \lambda<\infty, p(\cdot) \in \mathscr{B}\left(\mathbb{R}^{n}\right)$, and $\alpha(\cdot) \in L^{\infty}\left(\mathbb{R}^{n}\right)$ be log-Hölder continuous both at the origin and infinity, $2 \lambda \leq \alpha(\cdot), n \delta_{2} \leqslant \alpha(0), \alpha_{\infty}<\infty$, nonnegative integer and $s=\max \left\{\left[\alpha(0)-n \delta_{2}\right],\left[\alpha_{\infty}-n \delta_{2}\right]\right\}$, and $\delta_{2}$ as in Lemma 4 . If a sublinear operator $T$ satisfies that

(i) $T$ is bounded on $L^{p(\cdot)}$;

(ii) there exists a constant $\delta>0$ such that $s+\delta>$ $\max \left\{\alpha(0)-n \delta_{2}, \alpha_{\infty}-n \delta_{2}\right\}$, and for any compact support function $f$ with

$$
\int_{\mathbb{R}^{n}} f(x) x^{\beta} \mathrm{d} x=0, \quad|\beta| \leqslant s,
$$

Tf satisfies the size condition

$$
\begin{array}{r}
|T f(x)| \leqslant C(\operatorname{diam}(\operatorname{supp} f))^{s+\delta}|x|^{-(n+s+\delta)}\|f\|_{1}, \\
\text { when } \operatorname{dist}(x, \operatorname{supp} f) \geqslant \frac{|x|}{2} .
\end{array}
$$

Then there exists a constant $C$ such that

$$
\begin{aligned}
& \|T f\|_{M \dot{K}_{p(\cdot), \lambda}^{\alpha(\cdot), q}} \leqslant C\|f\|_{H M \dot{K}_{p(\cdot), \lambda}^{\alpha(\cdot), q},}, \\
& \|T f\|_{M K_{p(\cdot), \lambda}^{\alpha(\cdot), q}} \leqslant C\|f\|_{H M K_{p(\cdot), \lambda}^{\alpha(\cdot), q}}
\end{aligned}
$$

for $f \in H M \dot{K}_{p(\cdot), \lambda}^{\alpha(\cdot), q}$ and $f \in H M K_{p(\cdot), \lambda}^{\alpha(\cdot), q}$, respectively.

Proof. It suffices to prove the homogeneous case. Suppose $f \in H M \dot{K}_{p(\cdot), \lambda}^{\alpha(\cdot),}$. By Theorem 13, $f=\sum_{j=-\infty}^{\infty} \lambda_{j} b_{j}$ converges in $\delta^{\prime}\left(\mathbb{R}^{n}\right)$, where each $b_{j}$ is a central $(\alpha(\cdot), q(\cdot))$-atom with support contained in $B_{j}$ and

$$
\|f\|_{H M \dot{K}_{p(\cdot), \lambda}^{\alpha(\cdot), q}} \approx \inf \sup _{L \in \mathbb{Z}} 2^{-L \lambda}\left(\sum_{j=-\infty}^{L}\left|\lambda_{j}\right|^{q}\right)^{1 / q} .
$$

For simplicity, we denote $\Lambda=\sup _{L \in \mathbb{Z}^{2}} 2^{-L \lambda} \sum_{j=-\infty}^{L}\left|\lambda_{j}\right|^{q}$. By Proposition 7, we have

$$
\begin{array}{r}
\|T f\|_{M \dot{K}_{p(\cdot),}^{\alpha(), q}}^{q} \\
\approx \max \left\{\sup _{L \leqslant 0, L \in \mathbb{Z}} 2^{-L \lambda q} \sum_{k=-\infty}^{L} 2^{k q \alpha(0)}\left\|(T f) \chi_{k}\right\|_{L^{p(\cdot)}}^{q},\right. \\
\sup _{L>0, L \in \mathbb{Z}} 2^{-L \lambda q}\left(\sum_{k=-\infty}^{-1} 2^{k q \alpha(0)}\left\|(T f) \chi_{k}\right\|_{L^{p(\cdot)}}^{q}\right. \\
\left.\left.+\sum_{k=0}^{L} 2^{k q \alpha(\infty)}\left\|(T f) \chi_{k}\right\|_{L^{p(\cdot)}}^{q}\right)\right\}
\end{array}
$$

$\lesssim \max \{I, I I+I I I\}$

Thus, we finish the proof of Theorem 13. 
where

$$
\begin{gathered}
I:=\sup _{L \leqslant 0, L \in \mathbb{Z}} 2^{-L \lambda q} \sum_{k=-\infty}^{L} 2^{k q \alpha(0)}\left\|(T f) \chi_{k}\right\|_{L^{p()}}^{q}, \\
I I:=\sum_{k=-\infty}^{-1} 2^{k q \alpha(0)}\left\|(T f) \chi_{k}\right\|_{L^{p(\cdot)}}^{q}, \\
I I I:=\sup _{L>0, L \in \mathbb{Z}} 2^{-L \lambda q} \sum_{k=0}^{L} 2^{k q \alpha(\infty)}\left\|(T f) \chi_{k}\right\|_{L^{p(\cdot)}}^{q} .
\end{gathered}
$$

To complete our proof, we only need show that there exists a positive constant $C$ such that $I, I I, I I I \leqslant C \Lambda$.

First, we estimate $I$ :

$$
\begin{aligned}
I= & \sup _{L \leqslant 0, L \in \mathbb{Z}} 2^{-L \lambda q} \sum_{k=-\infty}^{L} 2^{k q \alpha(0)}\left\|(T f) \chi_{k}\right\|_{L^{p(\cdot)}}^{q} \\
\leqslant & \sup _{L \leqslant 0, L \in \mathbb{Z}} 2^{-L \lambda q} \sum_{k=-\infty}^{L} 2^{k q \alpha(0)}\left(\sum_{j=k}^{\infty}\left|\lambda_{j}\right|\left\|\left(T b_{j}\right) \chi_{k}\right\|_{L^{p(\cdot)}}\right)^{q} \\
& +\sup _{L \leqslant 0, L \in \mathbb{Z}} 2^{-L \lambda q} \sum_{k=-\infty}^{L} 2^{k q \alpha(0)}\left(\sum_{j=-\infty}^{k-1}\left|\lambda_{j}\right|\left\|\left(T b_{j}\right) \chi_{k}\right\|_{L^{p(\cdot)}}\right)^{q} \\
:= & I_{1}+I_{2} .
\end{aligned}
$$

By the boundedness of $T$ in $L^{p(\cdot)}$, we have

$$
\left\|\left(T b_{j}\right) \chi_{k}\right\|_{L^{p()}} \leqslant\left\|b_{j}\right\|_{L^{p()}} \leqslant\left|B_{j}\right|^{-\alpha_{j} / n}=2^{-\alpha_{j} j} .
$$

Therefore, when $0<q \leqslant 1$, we get

$$
\begin{aligned}
I_{1}= & \sup _{L \leqslant 0, L \in \mathbb{Z}} 2^{-L \lambda q} \sum_{k=-\infty}^{L} 2^{\alpha(0) k q}\left(\sum_{j=k}^{\infty}\left|\lambda_{j}\right|\left\|\left(T b_{j}\right) \chi_{k}\right\|_{L^{p(\cdot)}}\right)^{q} \\
& \leqslant \sup _{L \leqslant 0, L \in \mathbb{Z}} 2^{-L \lambda q} \sum_{k=-\infty}^{L} 2^{\alpha(0) k q}\left(\sum_{j=k}^{\infty}\left|\lambda_{j}\right| 2^{-\alpha_{j} j}\right)^{q} \\
\vdots & \sup _{L \leqslant 0, L \in \mathbb{Z}} 2^{-L \lambda q} \\
& \times \sum_{k=-\infty}^{L} 2^{\alpha(0) k q}\left(\sum_{j=k}^{-1}\left|\lambda_{j}\right|^{q} 2^{-\alpha(0) j q}+\sum_{j=0}^{\infty}\left|\lambda_{j}\right|^{q} 2^{-\alpha_{\infty} j q}\right) \\
\lesssim & \sup _{L \leqslant 0, L \in \mathbb{Z}} 2^{-L \lambda q} \sum_{k=-\infty}^{L} \sum_{j=k}^{-1}\left|\lambda_{j}\right|^{q} 2^{\alpha(0)(k-j) q} \\
& +\sup _{L \leqslant 0, L \in \mathbb{Z}} 2^{-L \lambda q} \sum_{k=-\infty}^{L} 2^{\alpha(0) k q} \sum_{j=0}^{\infty}\left|\lambda_{j}\right|^{q} 2^{-\alpha_{\infty} j q} \\
\vdots & \sup _{L \leqslant 0, L \in \mathbb{Z}} 2^{-L \lambda q} \sum_{j=-\infty}^{-1}\left|\lambda_{j}\right|^{q} \sum_{k=-\infty}^{j} 2^{\alpha(0)(k-j) q} \\
& +\sup _{L \leqslant 0, L \in \mathbb{Z} j=0}^{\infty} \sum_{j=0}^{-j \lambda q}\left|\lambda_{j}\right|^{q} 2^{\left(\lambda-\alpha_{\infty}\right) j q} 2^{-L \lambda q} \sum_{k=-\infty}^{L} 2^{\alpha(0) k q}
\end{aligned}
$$

$$
\begin{aligned}
\leqslant & \sup _{L \leqslant 0, L \in \mathbb{Z}} 2^{-L \lambda q} \sum_{j=-\infty}^{L}\left|\lambda_{j}\right|^{q} \\
& +\sup _{L \leqslant 0, L \in \mathbb{Z}} 2^{-L \lambda q} \sum_{j=L}^{-1}\left|\lambda_{j}\right|^{q} \sum_{k=-\infty}^{j} 2^{\alpha(0)(k-j) q} \\
& +\Lambda \sup _{L \leqslant 0, L \in \mathbb{Z}} \sum_{j=0}^{\infty} 2^{\left(\lambda-\alpha_{\infty}\right) j q} \sum_{k=-\infty}^{L} 2^{\alpha(0) k q-L \lambda q} \\
\leqslant & +\sup _{L \leqslant 0, L \in \mathbb{Z}} \sum_{j=L}^{-1} 2^{-j \lambda q}\left|\lambda_{j}\right|^{q} 2^{(j-L) \lambda q} \sum_{k=-\infty}^{j} 2^{\alpha(0)(k-j) q}+\Lambda \\
\leqslant & +\Lambda \sup _{L \leqslant 0, L \in \mathbb{Z}} \sum_{j=L}^{-1} 2^{(j-L) \lambda q} \sum_{k=-\infty}^{j} 2^{\alpha(0)(k-j) q} \\
\leqslant &
\end{aligned}
$$

When $1<q<\infty$, let $1 / q+1 / q^{\prime}=1$ and we obtain

$$
\begin{aligned}
& I_{1}=\sup _{L \leqslant 0, L \in \mathbb{Z}} 2^{-L \lambda q} \sum_{k=-\infty}^{L} 2^{\alpha(0) k q}\left(\sum_{j=k}^{\infty}\left|\lambda_{j}\right|\left\|\left(T b_{j}\right) \chi_{k}\right\|_{L^{p(\cdot)}}\right)^{q} \\
& \lesssim \sup _{L \leqslant 0, L \in \mathbb{Z}} 2^{-L \lambda q} \sum_{k=-\infty}^{L} 2^{\alpha(0) k q}\left(\sum_{j=k}^{\infty}\left|\lambda_{j}\right| 2^{-\alpha_{j} j}\right)^{q} \\
& \lesssim \sup _{L \leqslant 0, L \in \mathbb{Z}} 2^{-L \lambda q} \sum_{k=-\infty}^{L}\left(\sum_{j=k}^{-1}\left|\lambda_{j}\right| 2^{\alpha(0)(k-j)}\right)^{q} \\
& +\sup _{L \leqslant 0, L \in \mathbb{Z}} 2^{-L \lambda q} \sum_{k=-\infty}^{L} 2^{\alpha(0) k q}\left(\sum_{j=0}^{\infty}\left|\lambda_{j}\right| 2^{-\alpha_{\infty} j}\right)^{q} \\
& \lesssim \sup _{L \leqslant 0, L \in \mathbb{Z}} 2^{-L \lambda q} \sum_{k=-\infty}^{L}\left(\sum_{j=k}^{-1}\left|\lambda_{j}\right|^{q} 2^{\alpha(0)(k-j) q / 2}\right) \\
& \times\left(\sum_{j=k}^{-1} 2^{\alpha(0)(k-j) q^{\prime} / 2}\right)^{q / q^{\prime}} \\
& +\sup _{L \leqslant 0, L \in \mathbb{Z}} 2^{-L \lambda q} \sum_{k=-\infty}^{L} 2^{\alpha(0) k q}\left(\sum_{j=0}^{\infty}\left|\lambda_{j}\right|^{q} 2^{-\alpha_{\infty} j q / 2}\right) \\
& \times\left(\sum_{j=0}^{\infty} 2^{-\alpha_{\infty} j q^{\prime} / 2}\right)^{q / q^{\prime}} \\
& \lesssim \sup _{L \leqslant 0, L \in \mathbb{Z}} 2^{-L \lambda q} \sum_{k=-\infty}^{L} \sum_{j=k}^{-1}\left|\lambda_{j}\right|^{q} 2^{\alpha(0)(k-j) q / 2} \\
& +\sup _{L \leqslant 0, L \in \mathbb{Z}} 2^{-L \lambda q} \sum_{k=-\infty}^{L} 2^{\alpha(0) k q} \sum_{j=0}^{\infty}\left|\lambda_{j}\right|^{q} 2^{-\alpha_{\infty} j q / 2} \\
& \lesssim \sup _{L \leqslant 0, L \in \mathbb{Z}} 2^{-L \lambda q} \sum_{j=-\infty}^{-1}\left|\lambda_{j}\right|^{q} \sum_{k=-\infty}^{j} 2^{\alpha(0)(k-j) q / 2}
\end{aligned}
$$




$$
\begin{aligned}
& +\sup _{L \leqslant 0, L \in \mathbb{Z}} \sum_{j=0}^{\infty} 2^{-j \lambda q}\left|\lambda_{j}\right|^{q} 2^{\left(\lambda-\alpha_{\infty} / 2\right) j q} 2^{-L \lambda q} \sum_{k=-\infty}^{L} 2^{\alpha(0) k q} \\
& \leqslant \sup _{L \leqslant 0, L \in \mathbb{Z}} 2^{-L \lambda q} \sum_{j=-\infty}^{L}\left|\lambda_{j}\right|^{q} \\
& +\sup _{L \leqslant 0, L \in \mathbb{Z}} 2^{-L \lambda q} \sum_{j=L}^{-1}\left|\lambda_{j}\right|^{q} \sum_{k=-\infty}^{j} 2^{\alpha(0)(k-j) q / 2} \\
& +\Lambda \sup _{L \leqslant 0, L \in \mathbb{Z}} \sum_{j=0}^{\infty} 2^{\left(\lambda-\alpha_{\infty} / 2\right) j q} \sum_{k=-\infty}^{L} 2^{\alpha(0) k q-L \lambda q} \\
& \leqslant \Lambda+\sup _{L \leqslant 0, L \in \mathbb{Z}} \sum_{j=L}^{-1} 2^{-j \lambda q}\left|\lambda_{j}\right|^{q} 2^{(j-L) \lambda q} \sum_{k=-\infty}^{j} 2^{\alpha(0)(k-j) q / 2}+\Lambda \\
& \leqslant \Lambda+\Lambda \sup _{L \leqslant 0, L \in \mathbb{Z}} \sum_{j=L}^{-1} 2^{(j-L) \lambda q} \sum_{k=-\infty}^{j} 2^{\alpha(0)(k-j) q / 2} \\
& \leqslant \Lambda .
\end{aligned}
$$

So, we have $I_{1} \lesssim \Lambda$.

Second, we estimate $I_{2}$. By (71) and Lemma 3, we get

$$
\begin{aligned}
\left|T b_{j}(x)\right| & \lesssim|x|^{-(n+s+\delta)} 2^{j(s+\delta)} \int_{B_{j}}\left|b_{j}(y)\right| \mathrm{d} y \\
& \lesssim 2^{-k(n+s+\delta)} 2^{j(s+\delta)}\left\|b_{j}\right\|_{L^{p(\cdot)}}\left\|\chi_{B_{j}}\right\|_{L^{p^{\prime}(\cdot)}} \\
& \lesssim 2^{j\left(s+\delta-\alpha_{j}\right)-k(s+\delta+n)}\left\|\chi_{B_{j}}\right\|_{L^{p^{\prime}(\cdot)}} \cdot
\end{aligned}
$$

So by Lemmas 3 and 4, we have

$$
\begin{aligned}
& \left\|\left(T b_{j}\right) \chi_{k}\right\|_{L^{p(\cdot)}} \\
& \lesssim 2^{j\left(s+\delta-\alpha_{j}\right)-k(s+\delta+n)}\left\|\chi_{B_{j}}\right\|_{L^{p^{\prime}(\cdot)}}\left\|\chi_{B_{k}}\right\|_{L^{p(\cdot)}} \\
& \lesssim 2^{j\left(s+\delta-\alpha_{j}\right)-k(s+\delta)} 2^{-k n}\left(\left|B_{k}\right|\left\|\chi_{B_{k}}\right\|_{L^{p^{\prime}(\cdot)}}^{-1}\right)\left\|\chi_{B_{j}}\right\|_{L^{p^{\prime}(\cdot)}} \\
& \lesssim 2^{j\left(s+\delta-\alpha_{j}\right)-k(s+\delta)} \frac{\left\|\chi_{B_{j}}\right\|_{L^{p^{\prime}(\cdot)}}}{\left\|\chi_{B_{k}}\right\|_{L^{p^{\prime}(\cdot)}}} \\
& \lesssim 2^{\left(s+\delta+n \delta_{2}\right)(j-k)-j \alpha_{j}} .
\end{aligned}
$$

Therefore, when $0<q \leqslant 1$, by $n \delta_{2} \leqslant \alpha(0)<s+\delta+n \delta_{2}$ we get

$$
\begin{aligned}
I_{2}= & \sup _{L \leqslant 0, L \in \mathbb{Z}} 2^{-L \lambda q} \sum_{k=-\infty}^{L} 2^{\alpha(0) k q}\left(\sum_{j=-\infty}^{k-1}\left|\lambda_{j}\right|\left\|\left(T b_{j}\right) \chi_{k}\right\|_{L^{p(\cdot)}}\right)^{q} \\
\lesssim & \sup _{L \leqslant 0, L \in \mathbb{Z}} 2^{-L \lambda q} \\
& \times \sum_{k=-\infty}^{L} 2^{\alpha(0) k q}\left(\sum_{j=-\infty}^{k-1}\left|\lambda_{j}\right|^{q} 2^{\left[\left(s+\delta+n \delta_{2}\right)(j-k)-j \alpha(0)\right] q}\right)
\end{aligned}
$$

$$
\begin{aligned}
& =\sup _{L \leqslant 0, L \in \mathbb{Z}} 2^{-L \lambda q} \sum_{j=-\infty}^{L}\left|\lambda_{j}\right|^{q} \sum_{k=j+1}^{-1} 2^{(j-k)\left(s+\delta+n \delta_{2}-\alpha(0)\right) q} \\
& \lesssim \Lambda .
\end{aligned}
$$

When $1<q<\infty$, let $1 / q+1 / q^{\prime}=1$. Since $n \delta_{2} \leqslant \alpha(0)<$ $s+\delta+n \delta_{2}$, by Hölder's inequality, we have

$$
\begin{aligned}
I_{2}= & \sup _{L \leqslant 0, L \in \mathbb{Z}} 2^{-L \lambda q} \sum_{k=-\infty}^{L} 2^{\alpha(0) k q}\left(\sum_{j=-\infty}^{k-1}\left|\lambda_{j}\right|\left\|\left(T b_{j}\right) \chi_{k}\right\|_{L^{p(\cdot)}}\right)^{q} \\
\leqslant & \sup _{L \leqslant 0, L \in \mathbb{Z}} 2^{-L \lambda q} \sum_{k=-\infty}^{L} 2^{\alpha(0) k q}\left(\sum_{j=-\infty}^{k-1}\left|\lambda_{j}\right| 2^{\left(s+\delta+n \delta_{2}\right)(j-k)-j \alpha(0)}\right)^{q} \\
\lesssim & \sup _{L \leqslant 0, L \in \mathbb{Z}} 2^{-L \lambda q} \\
& \times \sum_{k=-\infty}^{L} 2^{\alpha(0) k q}\left(\sum_{j=-\infty}^{k-1}\left|\lambda_{j}\right|^{q} 2^{(j-k)\left(s+\delta+n \delta_{2}-\alpha(0)\right) q / 2}\right) \\
& \times\left(\sum_{j=-\infty}^{k-1} 2^{(j-k)\left(s+\delta+n \delta_{2}-\alpha(0)\right) q^{\prime} / 2}\right)^{q / q^{\prime}} \\
\lesssim & \sup _{L \leqslant 0, L \in \mathbb{Z}} 2^{-L \lambda q} \\
& \times \sum_{j=-\infty}^{L} 2^{\alpha(0) k q}\left(\sum_{j=-\infty}^{k-1}\left|\lambda_{j}\right|^{q} 2^{(j-k)\left(s+\delta+n \delta_{2}-\alpha(0)\right) q / 2}\right) \\
= & \sup _{L \leqslant 0, L \in \mathbb{Z}} 2^{-L \lambda q} \sum_{j=-\infty}^{L}\left|\lambda_{j}\right|^{q} \sum_{k=j+1}^{-1} 2^{(j-k)\left(s+\delta+n \delta_{2}-\alpha(0)\right) q / 2} \\
\leqslant & \Lambda .
\end{aligned}
$$

Hence, we have $I \lesssim \Lambda$.

Third, we estimate II. Consider

$$
\begin{aligned}
I I= & \sum_{k=-\infty}^{-1} 2^{k q \alpha(0)}\left\|(T f) \chi_{k}\right\|_{L^{p(\cdot)}}^{q} \\
\lesssim & \sum_{k=-\infty}^{-1} 2^{k q \alpha(0)}\left(\sum_{j=k}^{\infty}\left|\lambda_{j}\right|\left\|\left(T b_{j}\right) \chi_{k}\right\|_{L^{p(\cdot)}}\right)^{q} \\
& +\sum_{k=-\infty}^{-1} 2^{k q \alpha(0)}\left(\sum_{j=-\infty}^{k-1}\left|\lambda_{j}\right|\left\|\left(T b_{j}\right) \chi_{k}\right\|_{L^{p(\cdot)}}\right)^{q} \\
:= & I I_{1}+I I_{2} .
\end{aligned}
$$

When $0<q \leqslant 1$, we get

$$
\begin{aligned}
I I_{1} & =\sum_{k=-\infty}^{-1} 2^{\alpha(0) k q}\left(\sum_{j=k}^{\infty}\left|\lambda_{j}\right|\left\|\left(T b_{j}\right) \chi_{k}\right\|_{L^{p(\cdot)}}\right)^{q} \\
& \lesssim \sum_{k=-\infty}^{-1} 2^{\alpha(0) k q}\left(\sum_{j=k}^{\infty}\left|\lambda_{j}\right| 2^{-\alpha_{j} j}\right)^{q}
\end{aligned}
$$


$\lesssim \sum_{k=-\infty}^{-1} 2^{\alpha(0) k q}\left(\sum_{j=k}^{-1}\left|\lambda_{j}\right|^{q} 2^{-\alpha(0) j q}+\sum_{j=0}^{\infty}\left|\lambda_{j}\right|^{q} 2^{-\alpha_{\infty} j q}\right)$

$\lesssim \sum_{k=-\infty}^{-1} \sum_{j=k}^{-1}\left|\lambda_{j}\right|^{q} 2^{\alpha(0)(k-j) q}+\sum_{k=-\infty}^{-1} 2^{\alpha(0) k q} \sum_{j=0}^{\infty}\left|\lambda_{j}\right|^{q} 2^{-\alpha_{\infty} j q}$

$\lesssim \sum_{j=-\infty}^{-1}\left|\lambda_{j}\right|^{q} \sum_{k=-\infty}^{j} 2^{\alpha(0)(k-j) q}$

$+\sum_{j=0}^{\infty}\left|\lambda_{j}\right|^{q} 2^{-\alpha_{\infty} j q} \sum_{k=-\infty}^{-1} 2^{\alpha(0) k q}$

$\lesssim \sum_{j=-\infty}^{-1}\left|\lambda_{j}\right|^{q}+\sum_{j=0}^{\infty} 2^{-j \lambda q}\left|\lambda_{j}\right|^{q} 2^{-\alpha_{\infty} j q} \sum_{k=-\infty}^{-1} 2^{\alpha(0) k q}$

$\lesssim \Lambda+\Lambda \sum_{i=-\infty}^{j}\left|\lambda_{i}\right|^{q} \sum_{j=0}^{\infty} 2^{\left(\lambda-\alpha_{\infty}\right) j q} \sum_{k=-\infty}^{j} 2^{\alpha(0) k q}$

$\lesssim \Lambda$.

When $1<q<\infty$, let $1 / q+1 / q^{\prime}=1$ and we obtain

$$
\begin{aligned}
I I_{1}= & \sum_{k=-\infty}^{-1} 2^{\alpha(0) k q}\left(\sum_{j=k}^{\infty}\left|\lambda_{j}\right|\left\|\left(T b_{j}\right) \chi_{k}\right\|_{L^{p(\cdot)}}\right)^{q} \\
\leq & \sum_{k=-\infty}^{-1} 2^{\alpha(0) k q}\left(\sum_{j=k}^{\infty}\left|\lambda_{j}\right| 2^{-\alpha_{j} j}\right)^{q} \\
\leq & \sum_{k=-\infty}^{-1}\left(\sum_{j=k}^{-1}\left|\lambda_{j}\right| 2^{\alpha(0)(k-j)}\right)^{q} \\
& +\sum_{k=-\infty}^{-1} 2^{\alpha(0) k q}\left(\sum_{j=0}^{\infty}\left|\lambda_{j}\right| 2^{-\alpha_{\infty} j}\right)^{q} \\
\leq & \sum_{k=-\infty}^{-1}\left(\sum_{j=k}^{-1}\left|\lambda_{j}\right|^{q} 2^{\alpha(0)(k-j) q / 2}\right)\left(\sum_{j=k}^{-1} 2^{\alpha(0)(k-j) q^{\prime} / 2}\right)^{q / q^{\prime}} \\
& +\sum_{k=-\infty}^{-1} 2^{\alpha(0) k q}\left(\sum_{j=0}^{\infty}\left|\lambda_{j}\right|^{q} 2^{-\alpha_{\infty} j q / 2}\right)\left(\sum_{j=0}^{\infty} 2^{-\alpha_{\infty} j q^{\prime} / 2}\right)^{q / q^{\prime}} \\
\lesssim & \sum_{k=-\infty}^{-1}\left|\lambda_{j}\right|^{q} \sum_{k=-\infty}^{j} 2^{\alpha(0)(k-j) q / 2} \\
& +\sum_{j=0}^{\infty}\left|\lambda_{j}\right|^{q} 2^{-\alpha_{\infty} j q / 2} \sum_{k=-\infty}^{-1} 2^{\alpha(0) k q} \\
\leqslant & \sum_{j=-\infty}^{-1}\left|\lambda_{j}\right|^{q} \\
& +\sum_{j=0}^{\infty} 2^{\left(\lambda-\alpha_{\infty} / 2\right) j q} 2^{-j \lambda q} \sum_{i=-\infty}^{j}\left|\lambda_{i}\right|^{q} \sum_{k=-\infty}^{-1} 2^{\alpha(0) k q} \\
\leq & \Lambda \sum_{j=0}^{\infty} 2^{\left(\lambda-\alpha_{\infty} / 2\right) j q} \sum_{k=-\infty}^{-1} 2^{\alpha(0) k q} \\
&
\end{aligned}
$$

For $I I_{2}$, when $0<q \leqslant 1$, by $n \delta_{2} \leqslant \alpha(0)<s+\delta+n \delta_{2}$ we get

$$
\begin{aligned}
I I_{2} & =\sum_{k=-\infty}^{-1} 2^{\alpha(0) k q}\left(\sum_{j=-\infty}^{k-1}\left|\lambda_{j}\right|\left\|\left(T b_{j}\right) \chi_{k}\right\|_{L^{p(\cdot)}}\right)^{q} \\
& \leqslant \sum_{k=-\infty}^{-1} 2^{\alpha(0) k q}\left(\sum_{j=-\infty}^{k-1}\left|\lambda_{j}\right|^{q} 2^{\left[\left(s+\delta+n \delta_{2}\right)(j-k)-j \alpha(0)\right] q}\right) \\
& =\sum_{k=-\infty}^{-1}\left|\lambda_{j}\right|^{q} \sum_{k=j+1}^{-1} 2^{(j-k)\left(s+\delta+n \delta_{2}-\alpha(0)\right) q} \\
& \leqslant \sum_{k=-\infty}^{-1}\left|\lambda_{j}\right|^{q} \\
& \leqslant \Lambda .
\end{aligned}
$$

When $1<q<\infty$, let $1 / q+1 / q^{\prime}=1$. Since $n \delta_{2} \leqslant \alpha(0)<s+$ $\delta+n \delta_{2}$, by Hölder's inequality, we have

$$
\begin{aligned}
I I_{2} & =\sum_{k=-\infty}^{-1} 2^{\alpha(0) k q}\left(\sum_{j=-\infty}^{k-1}\left|\lambda_{j}\right|\left\|\left(T b_{j}\right) \chi_{k}\right\|_{L^{p \cdot(\cdot)}}\right)^{q} \\
& \leqslant \sum_{k=-\infty}^{-1} 2^{\alpha(0) k q}\left(\sum_{j=-\infty}^{k-1}\left|\lambda_{j}\right|^{\left(s+\delta+n \delta_{2}\right)(j-k)-j \alpha(0)}\right)^{q} \\
& \leqslant \sum_{k=-\infty}^{-1} 2^{\alpha(0) k q}\left(\sum_{j=-\infty}^{k-1}\left|\lambda_{j}\right|^{q} 2^{(j-k)\left(s+\delta+n \delta_{2}-\alpha(0)\right) q / 2}\right) \\
& \times\left(\sum_{j=-\infty}^{k-1} 2^{(j-k)\left(s+\delta+n \delta_{2}-\alpha(0)\right) q^{\prime} / 2}\right)^{q / q^{\prime}} \\
& \leqslant \sum_{j=-\infty}^{-1} 2^{\alpha(0) k q}\left(\sum_{j=-\infty}^{k-1}\left|\lambda_{j}\right|^{q} 2^{(j-k)\left(s+\delta+n \delta_{2}-\alpha(0)\right) q / 2}\right) \\
= & \sum_{j=-\infty}^{-1}\left|\lambda_{j}\right|^{q} \sum_{k=j+1}^{-1} 2^{(j-k)\left(s+\delta+n \delta_{2}-\alpha(0)\right) q / 2} \\
& \leqslant \Lambda .
\end{aligned}
$$

So, we have $I I \lesssim \Lambda$.

Finally, we estimate $I I I$ :

$$
\begin{aligned}
I I I= & \sup _{L>0, L \in \mathbb{Z}} 2^{-L \lambda q} \sum_{k=0}^{L} 2^{k q \alpha_{\infty}}\left\|(T f) \chi_{k}\right\|_{L^{p(\cdot)}}^{q} \\
\lesssim & \sup _{L>0, L \in \mathbb{Z}} 2^{-L \lambda q} \sum_{k=0}^{L} 2^{k q \alpha_{\infty}}\left(\sum_{j=k}^{\infty}\left|\lambda_{j}\right|\left\|\left(T b_{j}\right) \chi_{k}\right\|_{L^{p(\cdot)}}\right)^{q} \\
& +\sup _{L>0, L \in \mathbb{Z}} 2^{-L \lambda q} \sum_{k=0}^{L} 2^{k q \alpha_{\infty}}\left(\sum_{j=-\infty}^{k-1}\left|\lambda_{j}\right|\left\|\left(T b_{j}\right) \chi_{k}\right\|_{L^{p \cdot(\cdot)}}\right)^{q} \\
:= & I I I_{1}+I I I_{2} .
\end{aligned}
$$


When $0<q \leqslant 1$, by the boundedness of $T$ in $L^{p(\cdot)}$, we have

$$
\begin{aligned}
& I I I_{1}=\sup _{L>0, L \in \mathbb{Z}} 2^{-L \lambda q} \sum_{k=0}^{L} 2^{\alpha_{\infty} k q}\left(\sum_{j=k}^{\infty}\left|\lambda_{j}\right|\left\|\left(T b_{j}\right) \chi_{k}\right\|_{L^{p \cdot(\cdot)}}\right)^{q} \\
& \leqslant \sup _{L>0, L \in \mathbb{Z}} 2^{-L \lambda q} \sum_{k=0}^{L} 2^{\alpha_{\infty} k q} \sum_{j=k}^{\infty}\left|\lambda_{j}\right|^{q}\left\|\left(T b_{j}\right) \chi_{k}\right\|_{L^{p(\cdot)}}^{q} \\
& \lesssim \sup _{L>0, L \in \mathbb{Z}} 2^{-L \lambda q} \sum_{k=0}^{L} 2^{\alpha_{\infty} k q} \sum_{j=k}^{\infty}\left|\lambda_{j}\right|^{q} 2^{-\alpha_{j} j q} \\
& \lesssim \sup _{L>0, L \in \mathbb{Z}} 2^{-L \lambda q} \sum_{k=0}^{L} 2^{\alpha_{\infty} k q} \sum_{j=k}^{\infty}\left|\lambda_{j}\right|^{q} 2^{-\alpha_{\infty} j q} \\
& =\sup _{L>0, L \in \mathbb{Z}} 2^{-L \lambda q} \sum_{j=0}^{L}\left|\lambda_{j}\right|^{q} \sum_{k=0}^{j} 2^{(k-j) \alpha_{\infty} q} \\
& +\sup _{L>0, L \in \mathbb{Z}} 2^{-L \lambda q} \sum_{j=L}^{\infty}\left|\lambda_{j}\right|^{q} \sum_{k=0}^{L} 2^{(k-j) \alpha_{\infty} q} \\
& \lesssim \sup _{L>0, L \in \mathbb{Z}} 2^{-L \lambda q} \sum_{j=0}^{L}\left|\lambda_{j}\right|^{q} \\
& +\sup _{L>0, L \in \mathbb{Z}} \sum_{j=L}^{\infty} 2^{(j \lambda q-L \lambda q)} 2^{-j \lambda q} \sum_{i=-\infty}^{j}\left|\lambda_{i}\right|^{q} \sum_{k=0}^{L} 2^{(k-j) \alpha_{\infty} q} \\
& \lesssim \Lambda+\Lambda \sup _{L>0, L \in \mathbb{Z}} \sum_{j=L}^{\infty} 2^{(j-L) \lambda q} 2^{(L-j) \alpha_{\infty} q} \\
& \lesssim \Lambda+\Lambda \sup _{L>0, L \in \mathbb{Z}} \sum_{j=L}^{\infty} 2^{(j-L) q\left(\lambda-\alpha_{\infty}\right)} \\
& \lesssim \Lambda .
\end{aligned}
$$

When $1<q<\infty$, by the boundedness of $T$ in $L^{p(\cdot)}$ and Hölder's inequality, we have

$$
\begin{aligned}
I I I_{1}= & \sup _{L>0, L \in \mathbb{Z}} 2^{-L \lambda q} \sum_{k=0}^{L} 2^{\alpha_{\infty} k q}\left(\sum_{j=k}^{\infty}\left|\lambda_{j}\right|\left\|\left(T b_{j}\right) \chi_{k}\right\|_{L^{p(\cdot)}}\right)^{q} \\
\leqslant & \sup _{L>0, L \in \mathbb{Z}} 2^{-L \lambda q} \sum_{k=0}^{L} 2^{\alpha_{\infty} k q}\left(\sum_{j=k}^{\infty}\left|\lambda_{j}\right|^{q}\left\|\left(T b_{j}\right) \chi_{k}\right\|_{L^{p(.)}}^{q / 2}\right) \\
& \times\left(\sum_{j=k}^{\infty}\left\|\left(T b_{j}\right) \chi_{k}\right\|_{L^{p(\cdot)}}^{q^{\prime} / 2}\right)^{q / q^{\prime}} \\
\lesssim & \sup _{L>0, L \in \mathbb{Z}} 2^{-L \lambda q} \sum_{k=0}^{L} 2^{\alpha_{\infty} k q}\left(\sum_{j=k}^{\infty}\left|\lambda_{j}\right|^{q}\left\|b_{j}\right\|_{L^{p(\cdot)}}^{q / 2}\right) \\
& \times\left(\sum_{j=k}^{\infty}\left\|b_{j}\right\|_{L^{p}}^{\left.q^{\prime} / 2\right)}\right)^{q / q^{\prime}}
\end{aligned}
$$

$$
\begin{aligned}
& \lesssim \sup _{L>0, L \in \mathbb{Z}} 2^{-L \lambda q} \sum_{k=0}^{L} 2^{\alpha_{\infty} k q}\left(\sum_{j=k}^{\infty}\left|\lambda_{j}\right|^{q}\left|B_{j}\right|^{-\alpha_{j} q /(2 n)}\right) \\
& \times\left(\sum_{j=k}^{\infty}\left|B_{j}\right|^{-\alpha_{j} q^{\prime} /(2 n)}\right)^{q / q^{\prime}} \\
& \leqslant \sup _{L>0, L \in \mathbb{Z}} 2^{-L \lambda q} \sum_{k=0}^{L} 2^{\alpha_{\infty} k q / 2}\left(\sum_{j=k}^{\infty}\left|\lambda_{j}\right|^{q}\left|B_{j}\right|^{-\alpha_{j} q /(2 n)}\right) \\
& =\sup _{L>0, L \in \mathbb{Z}} 2^{-L \lambda q} \sum_{j=0}^{L}\left|\lambda_{j}\right|^{q} \sum_{k=0}^{j} 2^{(k-j) \alpha_{\infty} q / 2} \\
& +\sup _{L>0, L \in \mathbb{Z}} 2^{-L \lambda q} \sum_{j=L}^{\infty}\left|\lambda_{j}\right|^{q} \sum_{k=0}^{L} 2^{(k-j) \alpha_{\infty} q / 2} \\
& \leqslant \sup _{L>0, L \in \mathbb{Z}} 2^{-L \lambda q} \sum_{j=0}^{L}\left|\lambda_{j}\right|^{q} \\
& +\sup _{L>0, L \in \mathbb{Z}} \sum_{j=L}^{\infty} 2^{(j \lambda q-L \lambda q)} 2^{-j \lambda q} \sum_{i=-\infty}^{j}\left|\lambda_{i}\right|^{q} \sum_{k=0}^{L} 2^{(k-j) \alpha_{\infty} q / 2} \\
& \leq \Lambda+\Lambda \sup _{L>0, L \in \mathbb{Z}} \sum_{j=L}^{\infty} 2^{(j-L) \lambda q} 2^{(L-j) \alpha_{\infty} q / 2} \\
& \leqslant \Lambda+\Lambda \sup _{L>0, L \in \mathbb{Z}} \sum_{j=L}^{\infty} 2^{(j-L) q\left(\lambda-\alpha_{\infty} / 2\right)} \\
& \leq \Lambda .
\end{aligned}
$$

When $0<q \leqslant 1$, by $n \delta_{2} \leqslant \alpha(0), \alpha_{\infty}<s+\delta+n \delta_{2}$ we get

$$
\begin{aligned}
I I I_{2}= & \sup _{L>0, L \in \mathbb{Z}} 2^{-L \lambda q} \sum_{k=0}^{L} 2^{\alpha_{\infty} k q}\left(\sum_{j=-\infty}^{k-1}\left|\lambda_{j}\right|\left\|\left(T b_{j}\right) \chi_{k}\right\|_{L^{p(\cdot)}}\right)^{q} \\
\lesssim & \sup _{L>0, L \in \mathbb{Z}} 2^{-L \lambda q} \\
& \times \sum_{k=0}^{L} 2^{\alpha_{\infty} k q}\left(\sum_{j=-\infty}^{k-1}\left|\lambda_{j}\right|^{q} 2^{\left[\left(s+\delta+n \delta_{2}\right)(j-k)-j \alpha_{j}\right] q}\right) \\
= & \sup _{L>0, L \in \mathbb{Z}} 2^{-L \lambda q} \\
& \times \sum_{k=0}^{L} 2^{\alpha_{\infty} k q}\left(\sum_{j=-\infty}^{-1}\left|\lambda_{j}\right|^{q} 2^{\left[\left(s+\delta+n \delta_{2}\right)(j-k)-j \alpha(0)\right] q}\right) \\
& +\sup _{L>0, L \in \mathbb{Z}} 2^{-L \lambda q} \\
& \times \sum_{k=0}^{L} 2^{\alpha_{\infty} k q}\left(\sum_{j=0}^{k-1}\left|\lambda_{j}\right|^{q} 2^{\left[\left(s+\delta+n \delta_{2}\right)(j-k)-j \alpha_{\infty}\right] q}\right)
\end{aligned}
$$




$$
\begin{aligned}
& \lesssim \sup _{L>0, L \in \mathbb{Z}} 2^{-L \lambda q} \sum_{k=0}^{L} 2^{\left[\alpha_{\infty}-\left(s+\delta+n \delta_{2}\right)\right] k q} \\
& \quad \times \sum_{j=-\infty}^{-1}\left|\lambda_{j}\right|^{q} 2^{\left(s+\delta+n \delta_{2}-\alpha(0)\right) j q} \\
& +\sup _{L>0, L \in \mathbb{Z}} 2^{-L \lambda q} \sum_{k=0}^{L}\left|\lambda_{j}\right|^{q} \sum_{k=j+1}^{\infty} 2^{(j-k)\left(s+\delta+n \delta_{2}-\alpha_{\infty}\right) q} \\
& \lesssim \sup _{L>0, L \in \mathbb{Z}} 2^{-L \lambda q} \sum_{j=-\infty}^{-1}\left|\lambda_{j}\right|^{q}+\sup _{L>0, L \in \mathbb{Z}} 2^{-L \lambda q} \sum_{j=0}^{L-1}\left|\lambda_{j}\right|^{q}
\end{aligned}
$$$$
\lesssim \Lambda \text {. }
$$

When $1<q<\infty$, let $1 / q+1 / q^{\prime}=1$. Since $n \delta_{2} \leqslant \alpha(0)$, $\alpha_{\infty}<s+\delta+n \delta_{2}$, by Hölder's inequality, we have

$$
\begin{aligned}
& I I I_{2}=\sup _{L>0, L \in \mathbb{Z}} 2^{-L \lambda q} \sum_{k=0}^{L} 2^{\alpha_{\infty} k q}\left(\sum_{j=-\infty}^{k-1}\left|\lambda_{j}\right|\left\|\left(T b_{j}\right) \chi_{k}\right\|_{L^{p(\cdot)}}\right)^{q} \\
& \lesssim \sup _{L>0, L \in \mathbb{Z}} 2^{-L \lambda q} \sum_{k=0}^{L} 2^{\alpha_{\infty} k q}\left(\sum_{j=-\infty}^{k-1}\left|\lambda_{j}\right| 2^{\left[\left(s+\delta+n \delta_{2}\right)(j-k)-j \alpha_{j}\right]}\right)^{q} \\
& \lesssim \sup _{L>0, L \in \mathbb{Z}} 2^{-L \lambda q} \sum_{k=0}^{L} 2^{\alpha_{\infty} k q}\left(\sum_{j=-\infty}^{-1}\left|\lambda_{j}\right| 2^{\left[\left(s+\delta+n \delta_{2}\right)(j-k)-j \alpha(0)\right]}\right)^{q} \\
& +\sup _{L>0, L \in \mathbb{Z}} 2^{-L \lambda q} \sum_{k=0}^{L} 2^{\alpha_{\infty} k q}\left(\sum_{j=0}^{k-1}\left|\lambda_{j}\right| 2^{\left[\left(s+\delta+n \delta_{2}\right)(j-k)-j \alpha_{\infty}\right]}\right)^{q} \\
& \lesssim \sup _{L>0, L \in \mathbb{Z}} 2^{-L \lambda q} \sum_{k=0}^{L} 2^{\left[\alpha_{\infty}-\left(s+\delta+n \delta_{2}\right)\right] k q} \\
& \times\left(\sum_{j=-\infty}^{-1}\left|\lambda_{j}\right| 2^{\left(s+\delta+n \delta_{2}-\alpha(0)\right) j}\right)^{q} \\
& +\sup _{L>0, L \in \mathbb{Z}} 2^{-L \lambda q} \sum_{k=0}^{L}\left(\sum_{j=0}^{k-1}\left|\lambda_{j}\right| 2^{(j-k)\left(s+\delta+n \delta_{2}-\alpha_{\infty}\right)}\right)^{q} \\
& \lesssim\left(\sup _{L>0, L \in \mathbb{Z}} 2^{-L \lambda q} \sum_{j=-\infty}^{-1}\left|\lambda_{j}\right|^{q} 2^{\left(s+\delta+n \delta_{2}-\alpha(0)\right) j q / 2}\right) \\
& \times\left(\sum_{j=-\infty}^{-1} 2^{\left(s+\delta+n \delta_{2}-\alpha(0)\right) j q^{\prime} / 2}\right)^{q / q^{\prime}} \\
& +\sup _{L>0, L \in \mathbb{Z}} 2^{-L \lambda q} \sum_{k=0}^{L}\left(\sum_{j=0}^{k-1}\left|\lambda_{j}\right|^{q} 2^{(j-k)\left(s+\delta+n \delta_{2}-\alpha_{\infty}\right) q / 2}\right) \\
& \times\left(\sum_{j=0}^{k-1} 2^{(j-k)\left(s+\delta+n \delta_{2}-\alpha_{\infty}\right) q^{\prime} / 2}\right)^{q / q^{\prime}}
\end{aligned}
$$

$$
\begin{aligned}
& \lesssim \sup _{L>0, L \in \mathbb{Z}} 2^{-L \lambda q} \sum_{j=-\infty}^{-1}\left|\lambda_{j}\right|^{q} 2^{\left(s+\delta+n \delta_{2}-\alpha(0)\right) j q / 2} \\
& +\sup _{L>0, L \in \mathbb{Z}} 2^{-L \lambda q} \sum_{k=0}^{L} \sum_{j=0}^{k-1}\left|\lambda_{j}\right|^{q} 2^{(j-k)\left(s+\delta+n \delta_{2}-\alpha_{\infty}\right) q / 2} \\
& \lesssim \sup _{L>0, L \in \mathbb{Z}} 2^{-L \lambda q} \sum_{j=-\infty}^{-1}\left|\lambda_{j}\right|^{q} \\
& +\sup _{L>0, L \in \mathbb{Z}} 2^{-L \lambda q} \sum_{j=0}^{L-1}\left|\lambda_{j}\right|^{q} \sum_{k=j+1}^{L} 2^{(j-k)\left(s+\delta+n \delta_{2}-\alpha_{\infty}\right) q / 2} \\
& \leqslant \sup _{L>0, L \in \mathbb{Z}} 2^{-L \lambda q} \sum_{j=-\infty}^{-1}\left|\lambda_{j}\right|^{q}+\sup _{L>0, L \in \mathbb{Z}} 2^{-L \lambda q} \sum_{j=0}^{L-1}\left|\lambda_{j}\right|^{q}
\end{aligned}
$$

$\lesssim \Lambda$

Thus we have $I I I \lesssim \Lambda$. This finishes the proof of Theorem 14 .

Definition 15 . Let $K$ be a locally integrable function on $\mathbb{R}^{n} \times$ $\mathbb{R}^{n} \backslash\{x=y\}$. Then $K$ is called a standard kernel if there exist $\delta \in(0,1]$ and $C>0$, such that

$$
\begin{gathered}
|K(x, y)| \leqslant \frac{C}{|x-y|^{n}}, \quad x \neq y ; \\
\left|K(x, y)-K\left(x, y^{\prime}\right)\right| \leqslant C \frac{\left|y-y^{\prime}\right|^{\delta}}{|x-y|^{n+\delta}}, \\
\left|y-y^{\prime}\right| \leqslant \frac{1}{2}|x-y| ; \\
\left|K(x, y)-K\left(x^{\prime}, y\right)\right| \leqslant C \frac{\left|x-x^{\prime}\right|^{\delta}}{|x-y|^{n+\delta}} \\
\left|x-x^{\prime}\right| \leqslant \frac{1}{2}|x-y| .
\end{gathered}
$$

A linear operator $T: \mathcal{S}\left(\mathbb{R}^{n}\right) \rightarrow \mathcal{S}^{\prime}\left(\mathbb{R}^{n}\right)$ is called a Calderón-Zygmund operator associated to a standard kernel $K$ if

(i) $T$ can be extended to a bounded operator on $L^{2}\left(\mathbb{R}^{n}\right)$;

(ii) for any $f \in L^{2}$ with compact support and almost everywhere $x \notin \operatorname{supp} f$,

$$
T f(x)=\int_{\mathbb{R}^{n}} K(x, y) f(y) \mathrm{d} y .
$$

It is well known that a Calderón-Zygmund operator is also bounded in $L^{p(\cdot)}$ for any $p(\cdot) \in \mathscr{B}\left(\mathbb{R}^{n}\right)$; for example, see [38].

Corollary 16. Let $\alpha(\cdot)$ be a bounded and log-Hölder continuous both at the origin and infinity such that $n \delta_{2} \leqslant \alpha(0)$, 
$\alpha_{\infty}<n \delta_{2}+\delta$ with $\delta_{2}$ as in Lemma 4. Suppose T is a CalderónZygmund operator associated to a standard kernel $K$. If $p(\cdot) \in$ $\mathscr{B}\left(\mathbb{R}^{n}\right), 0<q<\infty$, and $0 \leq \lambda<\infty$, then there exists a constant $C$ such that

$$
\begin{gathered}
\|T f\|_{M \dot{K}_{p(\cdot), \lambda}^{\alpha(\cdot), q}} \leqslant C\|f\|_{H M \dot{K}_{p(\cdot), \lambda}^{\alpha(\cdot), q},}, \\
\|T f\|_{M K_{p(\cdot), \lambda}^{\alpha(\cdot), q}} \leqslant C\|f\|_{H M K_{p(\cdot), \lambda}^{\alpha(\cdot), q}}
\end{gathered}
$$

for $f \in H M \dot{K}_{p(\cdot), \lambda}^{\alpha(\cdot), q}$ and $f \in H M K_{p(\cdot), \lambda}^{\alpha(\cdot), q}$, respectively.

Proof. It is easy to know that $s=\max \left\{\left[\alpha(0)-n \delta_{2}\right],\left[\alpha_{\infty}-\right.\right.$ $\left.\left.n \delta_{2}\right]\right\}=0$ when $n \delta_{2} \leqslant \alpha(0)$ and $\alpha_{\infty}<n \delta_{2}+\delta$. Then the result follows from Theorem 14 .

Remark 17. For Hardy type spaces, there are some characterizations: maximal function, square function, atomic decomposition, and molecular decomposition. To discuss the boundedness of singular integrals in Hardy type spaces, we use the atomic decomposition for the domain Hardy space, while it is convenient to use another characterization of Hardy space for the target Hardy space; for example, see the proof of Theorem 6.7 .4 in [49] and [50, 51]. In a future paper, we will give the molecular decomposition of spaces $H M \dot{K}_{p(\cdot), \lambda}^{\alpha(\cdot), q}$ and $H M K_{p(\cdot), \lambda}^{\alpha(\cdot),}$. Then, by the atomic and molecular decompositions, we will obtain the boundedness of $T$ in Corollary 16 from $H M \dot{K}_{p(\cdot), \lambda}^{\alpha(\cdot), q}$ and $H M K_{p(\cdot), \lambda}^{\alpha(\cdot), q}$ into themselves, respectively.

\section{Conflict of Interests}

The authors declare that there is no conflict of interests regarding the publication of this paper.

\section{Acknowledgments}

This work was supported by the NSF of China (Grant no. 11361020). The authors express their thanks to the referee for the useful comments.

\section{References}

[1] A. Beurling, "Construction and analysis of some convolution algebras," Annales de L'Institut Fourier Grenoble, vol. 14, pp. 132, 1964.

[2] C. S. Herz, "Lipschitz spaces and Bernstein's theorem on absolutely convergent Fourier transforms," vol. 18, pp. 283-324, 1968.

[3] A. Baernstein II and E. Sawyer, " Embedding and multiplier theorems for $H^{p}\left(\mathbb{R}^{2}\right)$," Memoirs of the American Mathematical Society, vol. 59, no. 318, 1985.

[4] Y. Z. Chen and K.-S. Lau, "Some new classes of Hardy spaces," Journal of Functional Analysis, vol. 84, no. 2, pp. 255-278, 1989.

[5] J. García-Cuerva, "Hardy spaces and Beurling algebras," Journal of the London Mathematical Society, vol. 39, no. 3, pp. 499-513, 1989.

[6] S. Lu, D. Yang, and G. Hu, Herz Type Spaces and Their Applications, Science Press, Beijing, China, 2008.
[7] M. Ruzicka, Electrorheological Fluids: Modeling and Mathematical Theory, vol. 1748 of Lecture Notes in Mathematics, Springer, Berlin, Germany, 2000.

[8] P. Harjulehto, P. Hästö, Ú. V. Lê, and M. Nuortio, "Overview of differential equations with non-standard growth," Nonlinear Analysis: Theory, Methods \& Applications, vol. 72, no. 12, pp. 4551-4574, 2010.

[9] Y. Chen, S. Levine, and M. Rao, "Variable exponent, linear growth functionals in image restoration," SIAM Journal on Applied Mathematics, vol. 66, no. 4, pp. 1383-1406, 2006.

[10] F. Li, Z. Li, and L. Pi, "Variable exponent functionals in image restoration," Applied Mathematics and Computation, vol. 216, no. 3, pp. 870-882, 2010.

[11] P. Harjulehto, P. Hästö, V. Latvala, and O. Toivanen, "Critical variable exponent functionals in image restoration," Applied Mathematics Letters, vol. 26, no. 1, pp. 56-60, 2013.

[12] A. Almeida, J. Hasanov, and S. Samko, "Maximal and potential operators in variable exponent Morrey spaces," Georgian Mathematical Journal, vol. 15, no. 2, pp. 195-208, 2008.

[13] A. Almeida and P. Hästö, "Besov spaces with variable smoothness and integrability," Journal of Functional Analysis, vol. 258, no. 5, pp. 1628-1655, 2010.

[14] L. Diening, P. Hästö, and S. Roudenko, "Function spaces of variable smoothness and integrability," Journal of Functional Analysis, vol. 256, no. 6, pp. 1731-1768, 2009.

[15] B. Dong and J. Xu, "New Herz type Besov and Triebel-Lizorkin spaces with variable exponents," Journal of Function Spaces and Applications, vol. 2012, Article ID 384593, 27 pages, 2012.

[16] X. Fan, "Variable exponent Morrey and Campanato spaces," Nonlinear Analysis, vol. 72, no. 11, pp. 4148-4161, 2010.

[17] J.-J. Fu and J.-S. Xu, "Characterizations of Morrey type Besov and Triebel-Lizorkin spaces with variable exponents," Journal of Mathematical Analysis and Applications, vol. 381, no. 1, pp. 280-298, 2011.

[18] P. Gurka, P. Harjulehto, and A. Nekvinda, "Bessel potential spaces with variable exponent," Mathematical Inequalities \& Applications, vol. 10, no. 3, pp. 661-676, 2007.

[19] P. A. Hästö, "Local-to-global results in variable exponent spaces," Mathematical Research Letters, vol. 16, no. 2, pp. 263278, 2009.

[20] H. Kempka, "2-microlocal Besov and Triebel-Lizorkin spaces of variable integrability," Revista Matematica Complutense, vol. 22, no. 1, pp. 227-251, 2009.

[21] H. Kempka, "Atomic, molecular and wavelet decomposition of generalized 2-microlocal Besov spaces," Journal of Function Spaces and Applications, vol. 8, no. 2, pp. 129-165, 2010.

[22] E. Nakai and Y. Sawano, "Hardy spaces with variable exponents and generalized Campanato spaces," Journal of Functional Analysis, vol. 262, no. 9, pp. 3665-3748, 2012.

[23] J. Xu, "Variable Besov and Triebel-Lizorkin spaces," Annales Academice Scientiarum Fennica Mathematica, vol. 33, no. 2, pp. 511-522, 2008.

[24] J.-S. Xu, "An atomic decomposition of variable Besov and Triebel-Lizorkin spaces," Armenian Journal of Mathematics, vol. 2, no. 1, pp. 1-12, 2009.

[25] T. Noi, "Duality of variable exponent Triebel-Lizorkin and Besov spaces," Journal of Function Spaces and Applications, vol. 2012, Article ID 361807, 19 pages, 2012.

[26] Y. Sawano, "Atomic decompositions of Hardy spaces with variable exponents and its application to bounded linear operators," 
Integral Equations and Operator Theory, vol. 77, no. 1, pp. 123$148,2013$.

[27] M. Izuki, "Boundedness of sublinear operators on Herz spaces with variable exponent and application to wavelet characterization," Analysis Mathematica, vol. 36, no. 1, pp. 33-50, 2010.

[28] A. Almeida and D. Drihem, "Maximal, potential and singular type operators on Herz spaces with variable exponents," Journal of Mathematical Analysis and Applications, vol. 394, no. 2, pp. 781-795, 2012.

[29] C. Shi and J. Xu, "Herz type Besov and Triebel-Lizorkin spaces with variable exponent," Frontiers of Mathematics in China, vol. 8, no. 4, pp. 907-921, 2013.

[30] B. Dong and J. Xu, "New Herz type Besov and Triebel-Lizorkin spaces with variable exponents," Journal of Function Spaces and Applications, vol. 2012, Article ID 384593, 27 pages, 2012.

[31] H. Wang and Z. Liu, "The Herz-type Hardy spaces with variable exponent and their applications," Taiwanese Journal of Mathematics, vol. 16, no. 4, pp. 1363-1389, 2012.

[32] Z.-H. Xuan and L.-S. Shu, "Boundedness of higher order commutators on Herz-Morrey spaces with variable exponent," Journal of Nanjing University Mathematical Biquarterly, vol. 30, no. 2, pp. 188-196, 2013.

[33] Y. Lu and Y. P. Zhu, "Boundedness of multilinear CalderónZygmund singular operators on Morrey-Herz spaces with variable exponents," Acta Mathematica Sinica (English Series), vol. 30, no. 7, pp. 1180-1194, 2014.

[34] C. Tang, Q. Wu, and J. Xu, "Commutators of multilinear Calderón-Zygmund operator and BMO functions in HerzMorrey spaces with variable exponents," Journal of Function Spaces, vol. 2014, Article ID 162518, 12 pages, 2014.

[35] W. Gao, Y. Jiang, and X. Gong, "Boundedness of linear commutators on Herz-Morrey spaces on spaces of homogeneous type," Chinese Quarterly Journal of Mathematics, vol. 25, no. 2, pp. 172181, 2010.

[36] X. Q. Zhao and W. H. Gao, "Boundedness of sublinear operators on Herz-Morrey spaces," Applied Mathematics. A Journal of Chinese Universities. Series A, vol. 20, no. 1, pp. 55-62, 2005.

[37] D. V. Cruz-Uribe and A. Fiorenza, Variable Lebesgue Spaces, Applied and Numerical Harmonic Analysis, Birkhäuser, Heidelberg, Germany, 2013.

[38] D. Cruz-Uribe, A. Fiorenza, C. Martell, and C. Perez, "The Boundedness of classical operators on variable $L^{p}$ spaces," Annales Academiae Scientiarum Fennicae-Mathematica, vol. 31, pp. 239-264, 2006.

[39] D. Cruz-Uribe, A. Fiorenza, and C. J. Neugebauer, "The maximal function on variable $L^{p}$ spaces," Annales Academice Scientiarum Fennica Mathematica, vol. 28, no. 1, pp. 223-238, 2003.

[40] L. Diening, "Maximal function on generalized lebesgue spaces $L^{P(x)}$," Mathematical Inequalities and Applications, vol. 7, no. 2, pp. 245-253, 2004.

[41] L. Diening, P. Harjulehto, P. Hasto, and M. Ruzicka, Lebesgue and Sobolev Spaces with Variable Exponents, Springer, Berlin, Germany, 2011.

[42] A. Nekvinda, "Hardy-littlewood maxell operator on $L^{P(x)}$," Mathematical Inequalities and Applications, vol. 7, no. 2, pp. 255265, 2004.

[43] O. Kovacik and J. Rakosnik, "On spaces $L^{p(x)}$ and $W^{k, p(x), "}$ Czechoslovak Mathematical Journal, vol. 41, no. 4, pp. 592-681, 1991.
[44] C. Fefferman and E. M. Stein, " $H^{P}$ spaces of several variables," Acta Mathematica, vol. 129, no. 1, pp. 137-193, 1972.

[45] L. Grafakos, Classical Fourier Analysis, Springer, New York, NY, USA, 2008.

[46] E. M. Stein, Harmonic Analysis, Princeton University Press, Princeton, NJ, USA, 1993.

[47] A. Torchinsky, Real-Variable Methods in Harmonic Analysis, Academic Press, New York, NY, USA, 1986.

[48] S. Lu and D. Yang, "The local versions of $H^{p}\left(\mathbb{R}^{n}\right)$ spaces at the origin," Studia Mathematica, vol. 116, pp. 103-131, 1995.

[49] L. Grafakos, Modern Fourier Analysis, Springer, New York, NY, USA, 2009

[50] M. H. Taibleson and G. Weiss, "The molecular characterization of certain Hardy spaces," Astérisque, vol. 77, pp. 67-149, 1980.

[51] P. Rocha and M. Urciuolo, "Fractional type integral operators on variable Hardy spaces," Acta Mathematica Hungarica, vol. 143, no. 2, pp. 502-514, 2014. 


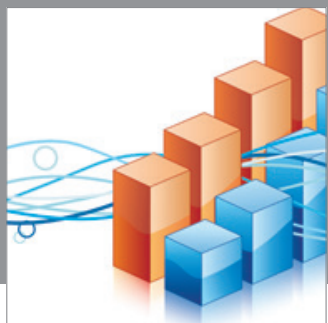

Advances in

Operations Research

mansans

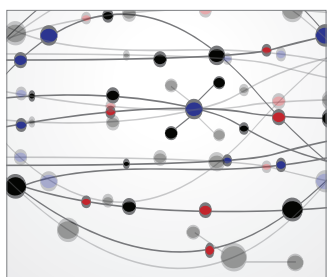

The Scientific World Journal
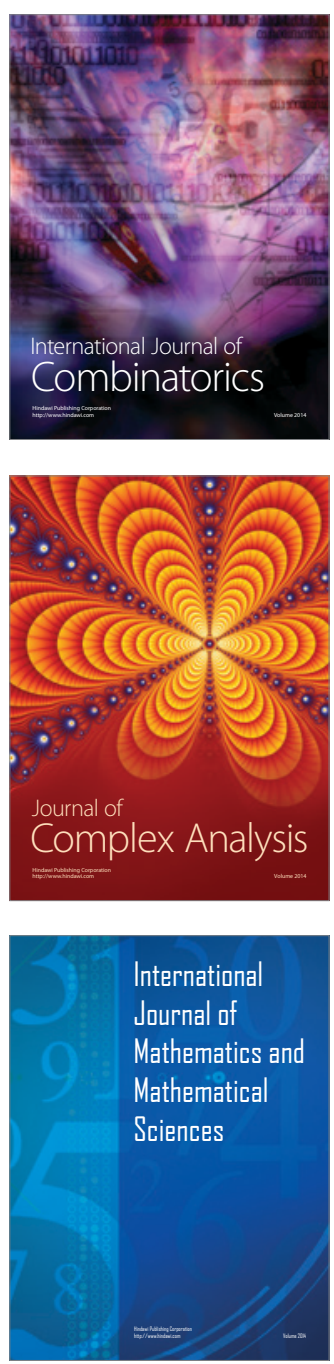
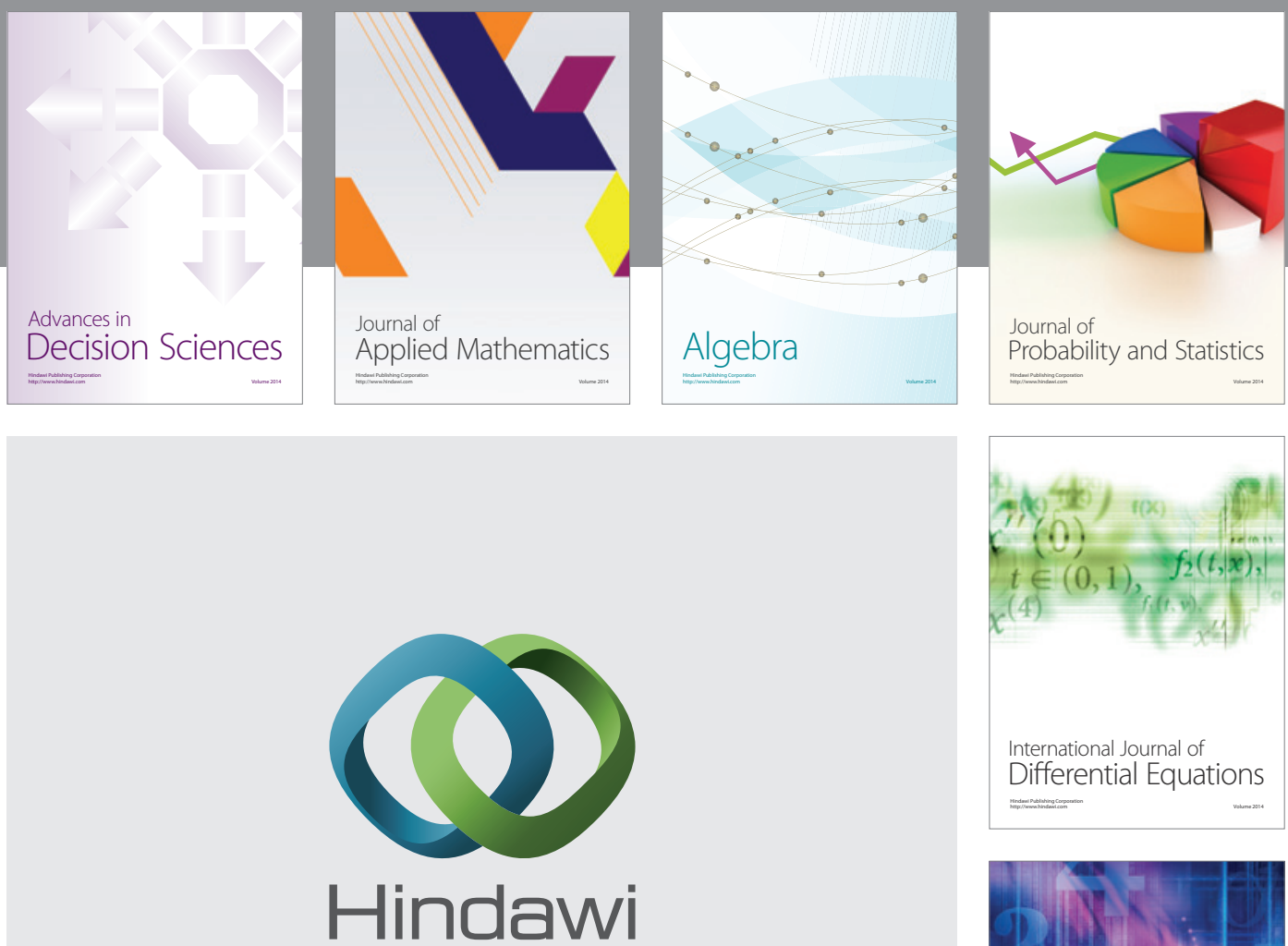

Submit your manuscripts at http://www.hindawi.com
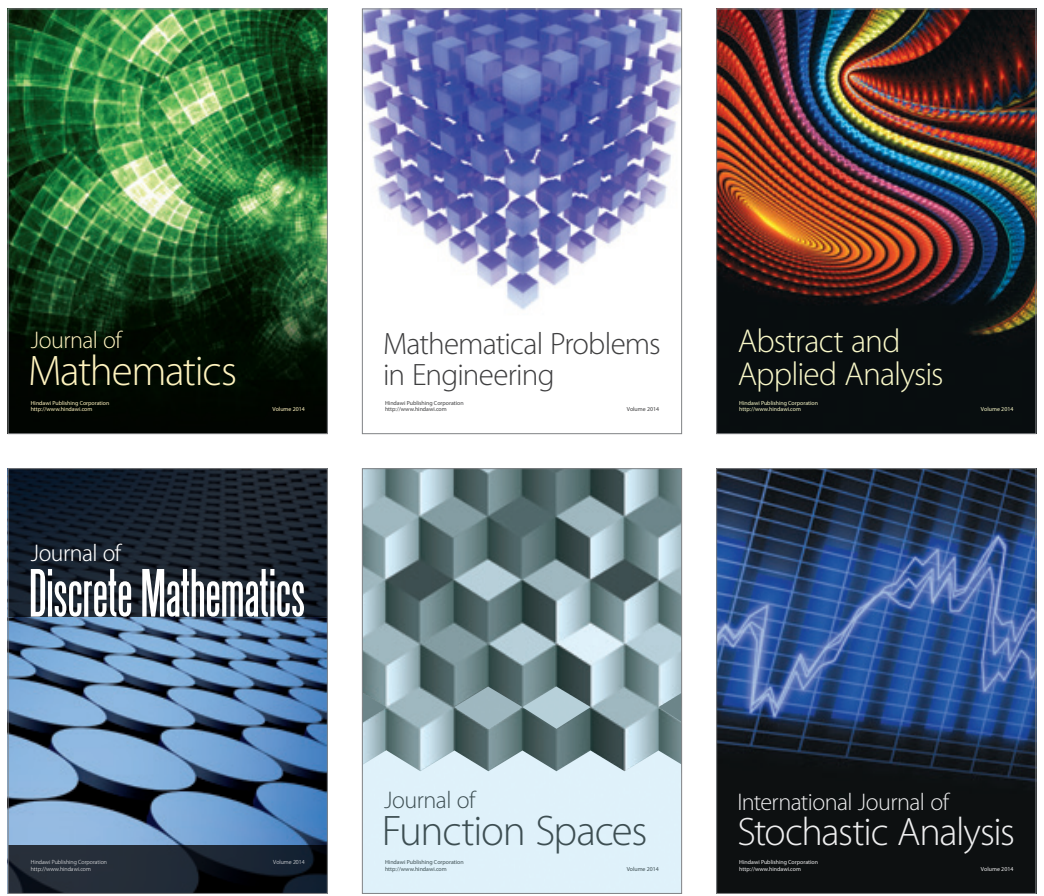

Journal of

Function Spaces

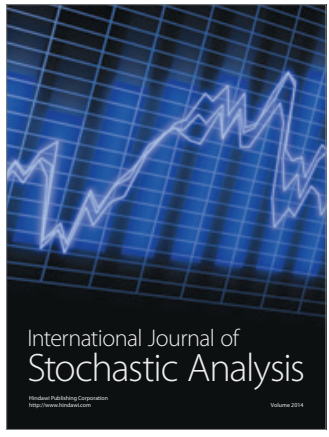

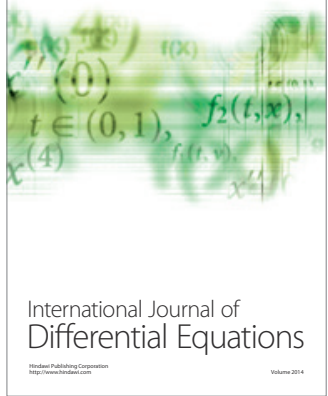
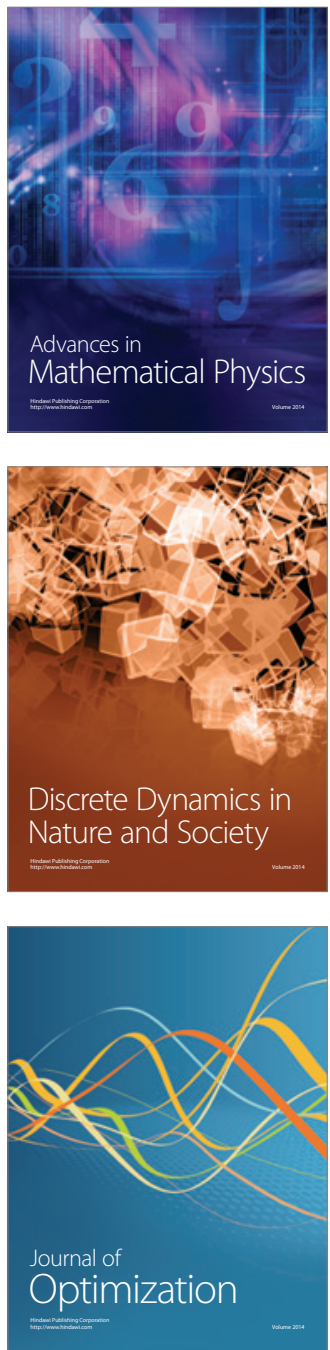